https://helda.helsinki.fi

\title{
Vibrational coordinates and their gradients : A geometric algebra approach
}

\section{Pesonen, Janne}

American Institute of Physics

2000-01-15

Journal of Chemical Physics -- February 15, 2000 -- Volume 112, Issue 7, pp. 3121-3132

http://link.aip.org/link/?JCP/112/3121/1

Downloaded from Helda, University of Helsinki institutional repository.

This is an electronic reprint of the original article.

This reprint may differ from the original in pagination and typographic detail.

Please cite the original version. 


\title{
Vibrational coordinates and their gradients: A geometric algebra approach
}

\author{
Janne Pesonen \\ Laboratory of Physical Chemistry, P.O. Box 55 (A. I. Virtasen Aukio 1), FIN-00014 University of Helsinki, \\ Helsinki, Finland
}

(Received 19 July 1999; accepted 16 November 1999)

\begin{abstract}
The gradients of vibrational coordinates are needed in order to form the exact vibrational kinetic energy operator of a polyatomic molecule. The conventional methods used to obtain these gradients are often quite laborious. However, by the methods of geometric algebra, the gradients for any vibrational coordinate can be easily calculated. Examples are given, and special attention is directed to ring coordinates. (C) 2000 American Institute of Physics. [S0021-9606(00)01106-5]
\end{abstract}

\section{INTRODUCTION}

In the past, the molecular vibrations were commonly studied by using the rectilinear normal coordinates based on the infinitesimal approximation of the true nuclear displacements. ${ }^{1,2}$ However, the rectilinear coordinates are not the best choice for the systems involving large displacements from the equilibrium configuration. Hence, in the recent years, much work has been done using the true curvilinear internal coordinates. For some recent work, see Refs. 3-11; for extensive bibliography, see Ref. 3 .

The use of curvilinear internal coordinates offers several advantages compared to the use of rectilinear normal coordinates. First, curvilinear internal coordinates must be used, if one wishes to take the advantage of the isotope-invariant Born-Oppenheimer potential energy surfaces. ${ }^{7,9}$ Second, potential energy surface expansions converge usually faster when expressed in terms of the curvilinear internal coordinates than when written in terms of rectilinear normal coordinates. ${ }^{12}$ This is especially true, if large amplitude motions ${ }^{10,11}$ are present. Last, but not least, the internal coordinates offer a simple and physical picture of vibrational motions in question. ${ }^{13-16}$ For example, in the curvilinear perspective, the dominant contributions to the nonlinear Fermi resonance arise from the anharmonic cubic kinetic energy and potential energy couplings. ${ }^{8,13}$ This is expected, since the Fermi resonance couples the vibrational modes of the 2:1 frequency ratio. In the rectilinear perspective, however, a major component to the Fermi resonance is due to the harmonic force constants associated with the exact internal coordinates. $^{13}$

The kinetic energy operator is more complicated in curvilinear internal coordinates than in rectilinear coordinates. The purpose of this work is to present a simple algebraic way to obtain the gradients of the vibrational coordinates, whose inner products give the exact kinetic energy operator.

\section{VIBRATIONAL KINETIC ENERGY}

By using the suitable internal coordinates $q_{i}$, the expectation value of the vibrational part of the kinetic energy of an $\mathrm{N}$-atom molecule,

$$
\langle T\rangle=-\frac{\hbar^{2}}{2} \sum_{\alpha=1}^{N} \int d \tau \Psi * \frac{\nabla_{\alpha}^{2}}{m_{\alpha}} \Psi
$$

can be written as ${ }^{17,18}$

$$
\langle T\rangle=-\frac{\hbar^{2}}{2} \int d \tau \Psi^{*} \sum_{i j}\left(\frac{\partial}{\partial q_{i}}+\frac{1}{J} \frac{\partial J}{\partial q_{i}}\right) g^{\left(q_{i} q_{j}\right)} \frac{\partial}{\partial q_{j}} \Psi,
$$

where $\Psi$ is the eigenfunction of the full vibrational Hamiltonian, $m_{\alpha}$ is the mass of the nucleus $\alpha$, and $\boldsymbol{\nabla}_{\alpha}$ is the vector derivative (gradient) operator with respect to the spatial position vector $\mathbf{x}_{\alpha}$ of the nucleus $\alpha$ and

$$
g^{\left(q_{i} q_{j}\right)}=\sum_{\alpha}^{N} \frac{1}{m_{\alpha}}\left(\nabla_{\alpha} q_{i}\right) \cdot\left(\nabla_{\alpha} q_{j}\right)
$$

are the elements of the mass weighted reciprocal metric tensor, whose values at the reference configuration are the Wilson $g$-matrix elements. ${ }^{1}$ In terms of a fixed three-dimensional orthonormal basis $\left\{\mathbf{u}_{1}, \mathbf{u}_{2}, \mathbf{u}_{3}\right\}$ the gradient operator possesses a representation

$$
\boldsymbol{\nabla}_{\alpha}=\sum_{k}^{3} \mathbf{u}_{k} \frac{\partial}{\partial x_{\alpha_{k}}},
$$

where $x_{\alpha_{k}}=\mathbf{x}_{\alpha} \cdot \mathbf{u}_{k}$ is the $k$ th Cartesian coordinate of the position vector $\mathbf{x}_{\alpha}$. The volume element of the integration is $d \tau=J d q_{1} d q_{2} \ldots$, where $J$ is the functional determinant (the Jacobian) of the coordinate transformation. In the curvilinear case, the $g^{\left(q_{i} q_{j}\right)}$ elements are generally functions of the internal coordinates.

In order to form the vibrational kinetic energy operator, the gradients $\boldsymbol{\nabla}_{\alpha} q_{i}$ must be calculated. Unfortunately, this is not always easy with the conventional methods. It seems surprising that no practical algebraic method to calculate these gradients has been presented in the literature. However, there exists a branch of mathematics, geometric algebra, which enables one to obtain the gradients for every internal coordinate both easily, straightforwardly and most surprisingly, coordinate freely. ${ }^{19-24}$

\section{RUDIMENTS OF GEOMETRIC ALGEBRA}

Physics today is blended with different mathematical formalisms, each introduced to handle some specific prob- 
lems. For example, the classical electrodynamics is commonly formulated in terms of the vector algebra developed by Gibbs, but on the other hand, the classical mechanics of the vibrating $N$-body system is formulated more readily by the matrix algebra. In some other cases, such as in the classical treatment of the forced linear oscillator, the complex algebra is more appropriate. Geometric algebra integrates all these algebraic systems in a coherent mathematical language, which retains the advantages of each of these subalgebras, but also possesses powerful new capabilities.

In the geometric algebra, an invertible geometric product is defined. Hence vectors can be divided and multiplied by other vectors (or any elements by any other elements for that matter) so they can be directly manipulated instead of manipulating their components. The geometric product for arbitrary vectors $\mathbf{a}$ and $\mathbf{b}$ is defined as $19,21,23$

$$
\mathbf{a b}=\mathbf{a} \cdot \mathbf{b}+\mathbf{a} \wedge \mathbf{b},
$$

where $\mathbf{a} \cdot \mathbf{b}$ is the scalar valued inner product (the usual "dot product"'), and $\mathbf{a} \wedge \mathbf{b}$ is a bivector (two-blade) valued outer product. The outer and inner products of two vectors can be vice versa expressed in terms of the geometric product only as

$$
\begin{aligned}
& \mathbf{a} \cdot \mathbf{b}=\frac{1}{2}(\mathbf{a b}+\mathbf{b a})=\mathbf{b} \cdot \mathbf{a}, \\
& \mathbf{a} \wedge \mathbf{b}=\frac{1}{2}(\mathbf{a b}-\mathbf{b a})=-\mathbf{b} \wedge \mathbf{a} .
\end{aligned}
$$

As seen from Eqs. (6) and (7), generally

$$
\mathbf{a b} \neq \mathbf{b a},
$$

and they are equal only if $\mathbf{a}$ is collinear with $\mathbf{b}$. However, the geometric product is both distributive and associative, i.e.,

$$
\begin{aligned}
& \mathbf{a}(\mathbf{b}+\mathbf{c})=\mathbf{a b}+\mathbf{a c}, \\
& \mathbf{a b c}=\mathbf{a}(\mathbf{b c})=(\mathbf{a b}) \mathbf{c} .
\end{aligned}
$$

The inverse of the vector $\mathbf{a}$ is given by

$$
\mathbf{a}^{-1}=\frac{\mathbf{a}}{a^{2}}
$$

and it fulfills

$$
\mathbf{a a}^{-1}=\mathbf{a}^{-1} \mathbf{a}=1
$$

as seen by the direct substitution of $\mathbf{b}=\mathbf{a} / a^{2}$ to Eq. (5).

The bivector $\mathbf{a}_{1} \wedge \mathbf{a}_{2}$ of two vectors $\mathbf{a}_{1}$ and $\mathbf{a}_{2}$ can be pictured as an oriented parallelogram with sides $\mathbf{a}_{1}$ and $\mathbf{a}_{2}$. Similarly, a trivector $\mathbf{a}_{1} \wedge \mathbf{a}_{2} \wedge \mathbf{a}_{3}$ can be pictured as an oriented parallelepiped with sides $\mathbf{a}_{1}, \mathbf{a}_{2}$, and $\mathbf{a}_{3}$. The inner, outer, and geometric products can be generalized as ${ }^{20,21}$

$$
\begin{aligned}
& \mathbf{a} \cdot A_{\bar{k}}=\frac{1}{2}\left(\mathbf{a} A_{\bar{k}}-(-1)^{k} A_{\bar{k}} \mathbf{a}\right)=(-1)^{k+1} A_{\bar{k}} \cdot \mathbf{a}, \\
& \mathbf{a} \wedge A_{\bar{k}}=\frac{1}{2}\left(\mathbf{a} A_{\bar{k}}+(-1)^{k} A_{\bar{k}} \mathbf{a}\right)=(-1)^{k} A_{\bar{k}} \wedge \mathbf{a}, \\
& \mathbf{a} A_{\bar{k}}=\mathbf{a} \cdot A_{\bar{k}}+\mathbf{a} \wedge A_{\bar{k}}
\end{aligned}
$$

for a vector $\mathbf{a}$ and any $k$-blade $A_{\bar{k}}=\mathbf{a}_{1} \wedge \mathbf{a}_{2} \wedge \cdots \wedge \mathbf{a}_{k}$. The inner product $\mathbf{a} \cdot A_{\bar{k}}$ is a $k-1$ blade, and the outer product $\mathbf{a} \wedge A_{\bar{k}}$ is a $k+1$ blade. Note, that the geometric product of two blades $A_{\bar{k}}$ and $B_{\bar{l}}$ is generally not related by the formula analogous to Eq. (15) if both $k, l>1$. However, one can write

$$
\begin{aligned}
& A_{\bar{k}} \cdot B_{\bar{l}}=\left\langle A_{\bar{k}} B_{\bar{l}}\right\rangle_{|\overline{k-l}|} \text { if } \quad k, l>0, \\
& A_{\bar{k}} \cdot B_{\bar{l}}=0 \quad \text { if } \quad k=0 \quad \text { or } \quad l=0, \\
& A_{\bar{k}} \wedge B_{\bar{l}}=\left\langle A_{\bar{k}} B_{\bar{l}}\right\rangle_{\overline{k+l}},
\end{aligned}
$$

where $\left\langle A_{\bar{k}} B_{\bar{l}}\right\rangle_{\bar{m}}$ denotes the $m$-blade part of $A_{\bar{k}} B_{\bar{l}}$. Generally, the geometric product $A_{\bar{k}} B_{\bar{l}}$ results in the terms of intermediate grade from $|k-l|$ to $k+l$ in the steps of two, i.e.,

$$
A_{\bar{k}} B_{\bar{l}}=\sum_{m=0}^{(k+l-|k-l|) / 2}\left\langle A_{\bar{k}} B_{\bar{l}}\right\rangle_{\mid \overline{k-l \mid+2 m}} .
$$

Since Eqs. (13)-(15) are sufficient for the purpose of gradient calculations, the explicit formulas need not be given for the factors of the right-hand side of Eq. (19). However, the special case of Eq. (17) together with Eq. (19) assures that scalars (the 0-blades) commute with every other blades, and that

$$
\lambda A_{\bar{k}}=\lambda \wedge A_{\bar{k}}=A_{\bar{k}} \wedge \lambda=A_{\bar{k}} \lambda .
$$

The magnitude of any multivector $A$ is a positive scalar, ${ }^{19-21}$

$$
|A|=\left\langle A^{\dagger} A\right\rangle_{\overline{0}}^{1 / 2} \geqslant 0,
$$

where $\left(\mathbf{a}_{1} \wedge \mathbf{a}_{2} \wedge \cdots \wedge \mathbf{a}_{k}\right)^{\dagger}=\mathbf{a}_{k} \wedge \cdots \wedge \mathbf{a}_{2} \wedge \mathbf{a}_{1}$ and $\left\langle A^{\dagger} A\right\rangle_{\bar{k}}$ denotes the $k$-blade part of $A^{\dagger} A$. Generally, if

$$
A=A_{\overline{0}}+A_{\overline{1}}+A_{\overline{2}}+\cdots,
$$

the square of the magnitude of $A$ is given by the sum of the squares of its $k$-blade parts $(k=0,1,2, \ldots)$, i.e.,

$$
\begin{aligned}
|A|^{2} & =\left|A_{\overline{0}}\right|^{2}+\left|A_{\overline{1}}\right|^{2}+\left|A_{\overline{2}}\right|^{2}+\cdots \\
& =A_{\overline{0}}^{2}+A_{\overline{1}}^{\dagger} \cdot A_{\overline{1}}+A_{\overline{2}}^{\dagger} \cdot A_{\overline{2}}+\ldots
\end{aligned}
$$

By using the Laplace's expansion formula (A1) given in Appendix A, the inner products of the $k$-blade parts in (23) can be expanded in terms of their vector factors.

In the three-dimensional space, the outer product $\mathbf{a}_{1}$ $\wedge \mathbf{a}_{2} \wedge \cdots \wedge \mathbf{a}_{k}$ is zero for $k>3$, and any trivector can be expressed as a multiple of a unit trivector $i$. As implied by its name, the unit trivector $i$ is of the unit magnitude, i.e., $i^{\dagger} i$ $=1=|i|$. On the other hand, $i^{2}=-1$. Furthermore, in the three-dimensional space, the unit trivector commutes with all other elements of the algebra. Hence it is justifiable to say, that the unit trivector $i$ plays the role of the imaginary unit in the three-dimensional space. Also, the vector cross product $\mathbf{a}_{1} \times \mathbf{a}_{2}$ is related to the bivector $\mathbf{a}_{1} \wedge \mathbf{a}_{2}$ as

$$
\mathbf{a}_{1} \times \mathbf{a}_{2}=-i\left(\mathbf{a}_{1} \wedge \mathbf{a}_{2}\right) .
$$

This very rudimentary introduction to the geometric algebra has been quite formal. However, an unlimited number of geometrical relations can be extracted by the simple algebraic manipulation of the rules given above. For example, any vector a can be decomposed to the components parallel $\left(\mathbf{a}_{\|}\right)$and orthogonal $\left(\mathbf{a}_{\perp}\right)$ to some given vector $\mathbf{n}$ by simply multiplying it by $\mathbf{n n}^{-1}$. This results

$$
\mathbf{a n n}^{-1}=\frac{\mathbf{a n n}}{n^{2}}=\frac{(\mathbf{a n}) \mathbf{n}}{n^{2}}=\frac{1}{n^{2}}(\mathbf{a} \cdot \mathbf{n}+\mathbf{a} \bigwedge \mathbf{n}) \mathbf{n}=\mathbf{a}_{\|}+\mathbf{a}_{\perp} .
$$


Similarly, any vector a can be decomposed to the components parallel $\left(\mathbf{a}_{\|}\right)$and orthogonal $\left(\mathbf{a}_{\perp}\right)$ to some given plane $\mathbf{B}=\mathbf{n} \wedge \mathbf{m}$ as

$$
\begin{aligned}
& \mathbf{a}_{\|}=(\mathbf{a} \cdot \mathbf{B}) \mathbf{B}^{-1}, \\
& \mathbf{a}_{\perp}=(\mathbf{a} \wedge \mathbf{B}) \mathbf{B}^{-1} .
\end{aligned}
$$

In the geometric algebra, each geometrical point is represented by a vector, and any geometric quantity can be described in terms of its intrinsic properties alone, without introducing any external coordinate frames (see especially Chaps. 2-6 of Ref.19). This means that any internal coordinate can be written in terms of the relative position vectors of the nuclei only. Once this has been done, the gradients can be easily obtained by the methods of the geometric algebra. The effort required in part of the reader to master the basics of the geometric algebra is more than compensated by the simplifications in the gradient calculations.

To eliminate some brackets, I shall use in this work the convention that the inner and outer products have preference over adjacent geometric products, and the outer product has preference over the adjacent inner product. For example, $\mathbf{a b} \cdot \mathbf{c} \wedge \mathbf{d} \equiv \mathbf{a}[\mathbf{b} \cdot(\mathbf{c} \wedge \mathbf{d})] \neq(\mathbf{a b}) \cdot(\mathbf{c} \wedge \mathbf{d})$.

\section{GRADIENTS OF THE INTERNAL COORDINATES}

The starting point is the following simple yet useful fact: In the geometric algebra, the gradients $\boldsymbol{\nabla}_{\alpha} q_{i}$ are the vector derivatives of the coordinate $q_{i}$ with respect to the spatial position vector $\mathbf{x}_{\alpha},{ }^{21,22}$ that is,

$$
\nabla_{\alpha} q_{i} \equiv \partial_{\mathbf{x}_{\alpha}} q_{i} .
$$

Much of the gradient calculations resemble those of the usual scalar calculus. For example, the vector differentiation is distributive,

$$
\boldsymbol{\nabla}_{\alpha}(F+G)=\nabla_{\alpha} F+\nabla_{\alpha} G
$$

for any $F$ and $G$. If $\lambda=\lambda\left(\mathbf{x}_{\alpha}\right)$ is scalar valued function, then

$$
\boldsymbol{\nabla}_{\alpha}(\lambda G)=\left(\boldsymbol{\nabla}_{\alpha} \lambda\right) G+\lambda \boldsymbol{\nabla}_{\alpha} G .
$$

However, in the general case the gradient operator does not commute with multivectors, and the product rule must be written as

$$
\nabla_{\alpha}(F G)=\grave{\nabla}_{\alpha} \grave{F} G+\grave{\nabla}_{\alpha} F \grave{G}
$$

where the target of differentiation is implicated by the accents. To make the notation as unambiguous as possible, I follow the convention that the vector derivative $\nabla_{\alpha}$ differentiates the quantities of its immediate right only. For example,

$$
\begin{aligned}
& \nabla_{\alpha} F G H=\grave{\nabla}_{\alpha} \grave{F} G H, \\
& \nabla_{\alpha}(F G) H=\grave{\nabla}_{\alpha} \grave{F} G H+\grave{\nabla}_{\alpha} F \grave{G} H .
\end{aligned}
$$

Unlike in the conventional vector calculus, vector derivatives are defined for all elements of the algebra. As the above notation implies, the $\boldsymbol{\nabla}_{\alpha} F$ is interpreted as the geometric product of the $\nabla_{\alpha}$ with the $F$. Hence by using Eq. (15), the vector derivative can be written as

$$
\boldsymbol{\nabla}_{\alpha} F=\nabla_{\alpha} \cdot F+\nabla_{\alpha} \wedge F=\nabla_{\alpha} \cdot F+i \nabla_{\alpha} \times F,
$$

where the last form is restricted to three-dimensional space only. It follows from Eq. (34) that the vector derivative operator changes the grade of the object it operates on by \pm 1 . For example, the vector derivative of the scalar $\lambda\left(\mathbf{x}_{\alpha}\right)$ is a vector (because $\mathbf{a} \cdot \lambda \equiv 0$ for any scalar $\lambda$, so $\mathbf{a} \lambda=\mathbf{a} \wedge \lambda$ ), and the vector derivative of the vector $\mathbf{f}\left(\mathbf{x}_{\alpha}\right)$ is a scalar plus a bivector.

The derivatives of handful of simple functions (given in Appendix A) combined with the product and chain rule allow the evaluation of any internal coordinate gradient. Some of these most basic identities are easily derived ${ }^{21}$ by expressing $\boldsymbol{\nabla}_{\alpha}$ as in Eq. (4) and using

$$
\begin{aligned}
& \mathbf{u}_{k} \mathbf{u}_{j}=-\mathbf{u}_{j} \mathbf{u}_{k} \text { for } k \neq j, \\
& \mathbf{u}_{k}^{2}=1 .
\end{aligned}
$$

For example,

$$
\begin{aligned}
& \boldsymbol{\nabla}_{\alpha} \mathbf{x}_{\alpha}=\sum_{k, j}^{3} \mathbf{u}_{k} \mathbf{u}_{j} \frac{\partial x_{\alpha_{j}}}{\partial x_{\alpha_{k}}}=\sum_{k}^{3} \frac{\partial x_{\alpha_{k}}}{\partial x_{\alpha_{k}}}=3 \\
& \boldsymbol{\nabla}_{\alpha} \mathbf{a} \cdot \mathbf{x}_{\alpha}=\sum_{k}^{3} \mathbf{u}_{k}\left(\mathbf{a} \cdot \frac{\partial \mathbf{x}_{\alpha}}{\partial x_{\alpha_{k}}}\right)=\sum_{k}^{3} \mathbf{u}_{k} \mathbf{a} \cdot \mathbf{u}_{k}=\mathbf{a}=\nabla_{\alpha} \mathbf{x}_{\alpha} \cdot \mathbf{a}
\end{aligned}
$$

for any $\mathbf{a}$ independent of $\mathbf{x}_{\alpha}$. Due to the distribution rule in Eq. (29), these results generalize to those given in the Appendix A, namely,

$$
\boldsymbol{\nabla}_{\beta} \mathbf{r}_{\alpha \beta}=3=-\nabla_{\alpha} \mathbf{r}_{\alpha \beta}
$$

and

$$
\nabla_{\beta} \mathbf{a} \cdot \mathbf{r}_{\alpha \beta}=\mathbf{a}=-\nabla_{\alpha} \mathbf{a} \cdot \mathbf{r}_{\alpha \beta},
$$

where $\mathbf{r}_{\alpha \beta}=\mathbf{x}_{\beta}-\mathbf{x}_{\alpha}$. These simple results can be used to evaluate the vector derivatives of more complicated functions. For example, the product rule of Eq. (31) together with Eq. (38) can be used to evaluate

$$
\boldsymbol{\nabla}_{\beta} \mathbf{r}_{\alpha \beta}^{2}=\grave{\nabla}_{\beta} \grave{\mathbf{r}}_{\alpha \beta} \cdot \mathbf{r}_{\alpha \beta}+\grave{\nabla}_{\beta} \mathbf{r}_{\alpha \beta} \cdot \grave{\mathbf{r}}_{\alpha \beta}=2 \mathbf{r}_{\alpha \beta} .
$$

In the internal coordinate gradient calculations following chain rule is needed frequently:

$$
\partial_{\mathbf{x}} M(\lambda(\mathbf{x}))=\partial_{\mathbf{x}}(\lambda(\mathbf{x})) \frac{\partial M}{\partial \lambda},
$$

where $\lambda=\lambda(\mathbf{x})$ is a scalar valued function of the vector variable $\mathbf{x}$, and $M$ is a multivector function of the scalar argument $\lambda(\mathbf{x})$. This rule can also be used to obtain other vector derivatives. For example,

$$
\boldsymbol{\nabla}_{\beta} r_{\alpha \beta}^{2}=2 r_{\alpha \beta} \nabla_{\beta} r_{\alpha \beta},
$$

where $r_{\alpha \beta}=\left|\mathbf{r}_{\alpha \beta}\right|$. Because $\mathbf{r}_{\alpha \beta}^{2}=r_{\alpha \beta}^{2}$, it follows from Eqs. (41) and (43) that

$$
\nabla_{\beta} r_{\alpha \beta}=\frac{\mathbf{r}_{\alpha \beta}}{r_{\alpha \beta}} .
$$

More vector derivatives can be obtained by combining the chain rule in Eq. (42) together with the derivative in Eq. (44). For example, the vector derivative of $r_{\alpha \beta}^{k}$ for any integer $k$ is easily obtained as 


$$
\boldsymbol{\nabla}_{\beta} r_{\alpha \beta}^{k}=\left(\nabla_{\beta} r_{\alpha \beta}\right) k r_{\alpha \beta}^{k-1}=k \mathbf{r}_{\alpha \beta} r_{\alpha \beta}^{k-2}
$$

Similarly,

$$
\begin{aligned}
\boldsymbol{\nabla}_{\beta}\left(r_{\alpha \beta}^{k} \mathbf{r}_{\alpha \beta}\right) & =\left(\boldsymbol{\nabla}_{\beta} r_{\alpha \beta}^{k}\right) \mathbf{r}_{\alpha \beta}+r_{\alpha \beta}^{k}\left(\boldsymbol{\nabla}_{\beta} \mathbf{r}_{\alpha \beta}\right) \\
& =k \mathbf{r}_{\alpha \beta} r_{\alpha \beta}^{k-2} \mathbf{r}_{\alpha \beta}+r_{\alpha \beta}^{k} \nabla_{\beta} \mathbf{r}_{\alpha \beta}=(k+3) r_{\alpha \beta}^{k},
\end{aligned}
$$

where Eqs. (30) and (39) were used.

It is emphasized, that the gradients of the internal coordinates derived by geometric algebra are exact, not just some approximations in the limit of infinitesimal vibrations. The following properties of the internal coordinate gradients can be used to check the gradients derived: ${ }^{1}$

(1) The sum of the internal displacements must be zero (i.e., the internal displacements cannot translate the molecule), so

$$
\sum_{\alpha} \nabla_{\alpha} q_{i}=0
$$

(2) The internal displacements cannot rotate the molecule, so

$$
\sum_{\alpha} \mathbf{x}_{\alpha} \times \nabla_{\alpha} q_{i}=0
$$

\section{A. Some acyclic internal coordinates}

In this section, the gradients for two the most basic internal coordinates, bond stretching and valence angle bending are derived using geometric calculus. These are included to emphasize the elegancy and the effectiveness of the geometric algebra approach compared to other methods. Furthermore, I derive the complete gradients of the out-of-plane coordinate. Last, I define a new twisting coordinate and derive its gradients. All the calculations are presented in some detail to allow the reader to compare my method to other possible ways to obtain the gradients. It should be mentioned that geometric algebra was used earlier to define the inversion coordinate of ammonia and to derive its gradients. ${ }^{11}$

\section{Bond stretching}

The most basic of all possible internal coordinates is the bond length $r_{\alpha \beta}=\left|\mathbf{r}_{\alpha \beta}\right|$, where $\mathbf{r}_{\alpha \beta}=\mathbf{x}_{\beta}-\mathbf{x}_{\alpha}$ (see Fig. 1). The gradients are obtained by the simple vectorial differentiation,

$$
\begin{aligned}
& \partial_{\mathbf{x}_{\alpha}} r_{\alpha \beta}=-\mathbf{u}_{\mathbf{r}_{\alpha \beta}} \equiv \nabla_{\alpha} r_{\alpha \beta}, \\
& \partial_{\mathbf{x}_{\beta}} r_{\alpha \beta}=\mathbf{u}_{\mathbf{r}_{\alpha \beta}},
\end{aligned}
$$

where $\mathbf{u}_{\mathbf{r}_{\alpha \beta}}$ is a unit vector in the direction of $\mathbf{r}_{\alpha \beta}$ [see the derivation of Eq. (44)]. This is, of course, a familiar result. ${ }^{1,2}$

\section{Valence angle bending}

The valence angle $\theta_{\beta \alpha \gamma}$ (see Fig. 2) is defined via

$$
\mathbf{r}_{\alpha \beta} \cdot \mathbf{r}_{\alpha \gamma}=r_{\alpha \beta} r_{\alpha \gamma} \cos \theta_{\beta \alpha \gamma},
$$

where $\mathbf{r}_{\alpha \gamma}=\mathbf{x}_{\gamma}-\mathbf{x}_{\alpha}$ and, by construction, it is always the smaller of the two possible angles (i.e., $0 \leqslant \theta_{\beta \alpha \gamma} \leqslant \pi$ ). To relate a variation in the bond angle to the atomic positions

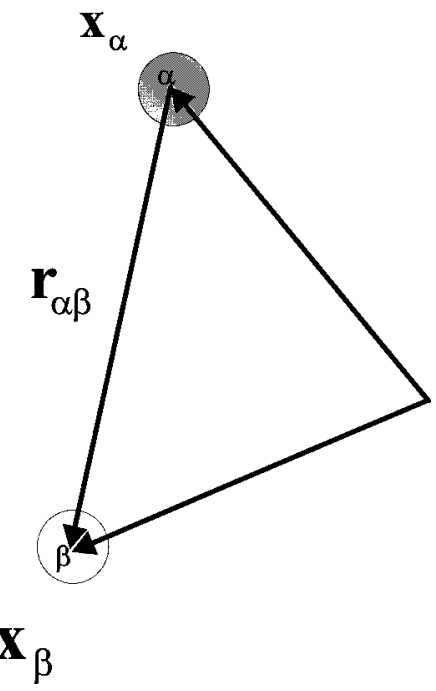

FIG. 1. Bond stretching $r_{\alpha \beta}=\left|\mathbf{r}_{\alpha \beta}\right|$.

$\mathbf{x}_{\alpha}, \mathbf{x}_{\beta}$, and $\mathbf{x}_{\gamma}$, both sides of Eq. (51) are needed. The gradient of the left-hand side is obtained with Eq. (40) as

$$
\partial_{\mathbf{x}_{\beta}}\left(\mathbf{r}_{\alpha \beta} \cdot \mathbf{r}_{\alpha \gamma}\right)=\mathbf{r}_{\alpha \gamma} .
$$

The right-hand side is differentiated as

$$
\begin{aligned}
\partial_{\mathbf{x}_{\beta}}\left(r_{\alpha \beta} r_{\alpha \gamma} \cos \theta_{\beta \alpha \gamma}\right)= & \left(\partial_{\mathbf{x}_{\beta}} r_{\alpha \beta}\right) r_{\alpha \gamma} \cos \theta_{\beta \alpha \gamma} \\
& +r_{\alpha \beta} r_{\alpha \gamma} \partial_{\mathbf{x}_{\beta}}\left(\cos \theta_{\beta \alpha \gamma}\right) \\
= & \mathbf{u}_{\mathbf{r}_{\alpha \beta}} r_{\alpha \gamma} \cos \theta_{\beta \alpha \gamma} \\
& -r_{\alpha \beta} r_{\alpha \gamma} \sin \theta_{\beta \alpha \gamma} \partial_{\mathbf{x}_{\beta}} \theta_{\beta \alpha \gamma},
\end{aligned}
$$

where the product rule of Eq. (30) and the chain rule of Eq. (42) were used to obtain the final result. The gradients are therefore

$$
\begin{aligned}
\partial_{\mathbf{x}_{\beta}} \theta_{\beta \alpha \gamma} & =\frac{\mathbf{u}_{\mathbf{r}_{\alpha \beta}} \cos \theta_{\beta \alpha \gamma}-\mathbf{u}_{\mathbf{r}_{\alpha \gamma}}}{r_{\alpha \beta} \sin \theta_{\beta \alpha \gamma}}, \\
\partial_{\mathbf{x}_{\gamma}} \theta_{\beta \alpha \gamma} & =\frac{\mathbf{u}_{\alpha \gamma} \cos \theta_{\beta \alpha \gamma}-\mathbf{u}_{\mathbf{r}_{\alpha \beta}}}{r_{\alpha \gamma} \sin \theta_{\beta \alpha \gamma}} .
\end{aligned}
$$

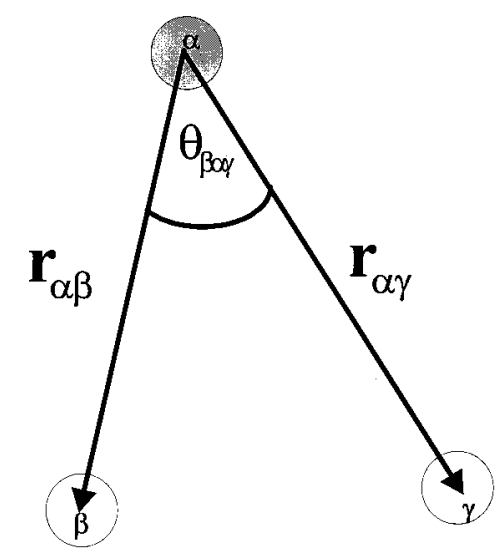

FIG. 2. Valence angle $\theta_{\beta \alpha \gamma}$ spanned by the bonds $\mathbf{r}_{\alpha \beta}$ and $\mathbf{r}_{\alpha \gamma}$. 


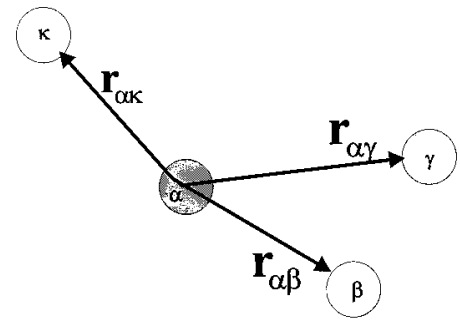

FIG. 3. Bond vectors $\mathbf{r}_{\alpha \beta}, \mathbf{r}_{\alpha \gamma}$, and $\mathbf{r}_{\alpha \kappa}$ in dextral order (and the value of out-of-plane coordinate $\varphi_{\alpha \beta \gamma \kappa}$ is positive).

The gradient of the central atom is obtained by direct differentiation as

$$
\partial_{\mathbf{x}_{\alpha}} \theta_{\beta \alpha \gamma}=-\left(\partial_{\mathbf{x}_{\beta}} \theta_{\beta \alpha \gamma}+\partial_{\mathbf{x}_{\gamma}} \theta_{\beta \alpha \gamma}\right)
$$

in accordance with Eq. (47). These results are identical to those given in Refs. 1,2.

\section{Out-of-plane bending}

The chiral out-of-plane bending coordinate $\varphi_{\alpha \beta \gamma \kappa}$ is defined as the signed volume of the parallelepiped spanned by the bonds $\mathbf{r}_{\alpha \beta}, \mathbf{r}_{\alpha \gamma}$ and $\mathbf{r}_{\alpha \kappa}$ divided by the lengths of these bonds, ${ }^{16}$ i.e.,

$$
\varphi_{\alpha \beta \gamma \kappa}=-i \frac{\mathbf{r}_{\alpha \beta} \wedge \mathbf{r}_{\alpha \gamma} \wedge \mathbf{r}_{\alpha \kappa}}{r_{\alpha \beta} r_{\alpha \gamma} r_{\alpha \kappa}},
$$

where $i$ is the dextral (right-handed) unit trivector. [One can write $\varphi_{\alpha \beta \gamma \kappa}=\left(\mathbf{r}_{\alpha \beta} \cdot \mathbf{r}_{\alpha \gamma} \times \mathbf{r}_{\alpha \kappa}\right) /\left(r_{\alpha \beta} r_{\alpha \gamma} r_{\alpha \kappa}\right)$ as well, since $\mathbf{r}_{\alpha \beta} \wedge \mathbf{r}_{\alpha \gamma} \wedge \mathbf{r}_{\alpha \kappa}=i \mathbf{r}_{\alpha \beta} \cdot \mathbf{r}_{\alpha \gamma} \times \mathbf{r}_{\alpha \kappa}$.] If the dextral order of the bond vectors is fixed as $\left\{\mathbf{r}_{\alpha \beta}, \mathbf{r}_{\alpha \gamma}, \mathbf{r}_{\alpha \kappa}\right\}$, the positive sign of $\varphi_{\alpha \beta \gamma \kappa}$ means that the bond vectors form a right-handed set (and the molecule has bent "up;" see Fig. 3). Likewise, if the sign of $\varphi_{\alpha \beta \gamma \kappa}$ is negative, the bond vectors form a lefthanded set (and the molecule has bent "down"). The gradients of the out-of-plane coordinate are easily obtained by directly differentiating the both sides of Eq. (57) with respect to the atomic positions. This results in

$$
\begin{aligned}
\partial_{\mathbf{x}_{\beta}} \varphi_{\alpha \beta \gamma \kappa}= & -i\left[\frac{1}{r_{\alpha \beta} r_{\alpha \gamma} r_{\alpha \kappa}} \partial_{\mathbf{x}_{\beta}} \mathbf{r}_{\alpha \beta} \wedge \mathbf{r}_{\alpha \gamma} \wedge \mathbf{r}_{\alpha \kappa}\right. \\
& \left.+\left(\partial_{\mathbf{x}_{\beta}} \frac{1}{r_{\alpha \beta} r_{\alpha \gamma} r_{\alpha \kappa}}\right) \mathbf{r}_{\alpha \beta} \wedge \mathbf{r}_{\alpha \gamma} \wedge \mathbf{r}_{\alpha \kappa}\right] \\
= & \frac{\mathbf{r}_{\alpha \gamma} \times \mathbf{r}_{\alpha \kappa}}{r_{\alpha \beta} r_{\alpha \gamma} r_{\alpha \kappa}}+i \frac{\left(\partial_{\mathbf{x}_{\beta}} r_{\alpha \beta}\right) \mathbf{r}_{\alpha \beta} \wedge \mathbf{r}_{\alpha \gamma} \wedge \mathbf{r}_{\alpha \kappa}}{r_{\alpha \beta}^{2} r_{\alpha \gamma} r_{\alpha \kappa}} \\
= & \frac{\mathbf{r}_{\alpha \gamma} \times \mathbf{r}_{\alpha \kappa}}{r_{\alpha \beta} r_{\alpha \gamma} r_{\alpha \kappa}}-\varphi_{\alpha \beta \gamma \kappa} \frac{\mathbf{r}_{\alpha \beta}}{r_{\alpha \beta}^{2}},
\end{aligned}
$$

where the identity

$$
-i \partial_{\mathbf{x}_{\beta}}\left(\mathbf{r}_{\alpha \beta} \wedge \mathbf{r}_{\alpha \gamma} \wedge \mathbf{r}_{\alpha \kappa}\right)=-i \mathbf{r}_{\alpha \gamma} \wedge \mathbf{r}_{\alpha \kappa}=\mathbf{r}_{\alpha \gamma} \times \mathbf{r}_{\alpha \kappa}
$$

was used (see Appendix A). The gradients $\partial_{\mathbf{x}_{\gamma}} \varphi_{\alpha \beta \gamma \kappa}$ and $\partial_{\mathbf{x}_{\kappa}} \varphi_{\alpha \beta \gamma \kappa}$ are obtained by cyclically permuting indices $\beta, \gamma$, and $\kappa$, and they are given in Table I. The central atom gradient is given by

$$
\partial_{\mathbf{x}_{\alpha}} \varphi_{\alpha \beta \gamma \kappa}=-\left(\partial_{\mathbf{x}_{\beta}} \varphi_{\alpha \beta \gamma \kappa}+\partial_{\mathbf{x}_{\gamma}} \varphi_{\alpha \beta \gamma \kappa}+\partial_{\mathbf{x}_{\kappa}} \varphi_{\alpha \beta \gamma \kappa}\right) .
$$

\section{Twisting}

I define the twisting coordinate $\xi_{\alpha \beta \gamma \kappa \lambda}$ as the dihedral angle ${ }^{20}$ between the planes $\mathbf{r}_{\alpha \beta} \wedge \mathbf{r}_{\alpha \gamma}$ and $\mathbf{r}_{\alpha \kappa} \wedge \mathbf{r}_{\alpha \lambda}$ (Fig. 4). In terms of the unit bond vectors,

$$
\cos \xi_{\alpha \beta \gamma \kappa \lambda}=\frac{\left(\mathbf{u}_{\mathbf{r}_{\alpha \beta}} \wedge \mathbf{u}_{\mathbf{r}_{\alpha \gamma}}\right)^{\dagger}}{\left|\mathbf{u}_{\mathbf{r}_{\alpha \beta}} \wedge \mathbf{u}_{\mathbf{r}_{\alpha \gamma}}\right|} \cdot \frac{\mathbf{u}_{\mathbf{r}_{\alpha \kappa}} \wedge \mathbf{u}_{\mathbf{r}_{\alpha \lambda}}}{\left|\mathbf{u}_{\mathbf{r}_{\alpha \kappa}} \wedge \mathbf{u}_{\mathbf{r}_{\alpha \lambda}}\right|}
$$

$$
\begin{aligned}
& =\frac{\mathbf{u}_{\mathbf{r}_{\alpha \gamma}} \wedge \mathbf{u}_{\mathbf{r}_{\alpha \beta}}}{\left|\mathbf{u}_{\mathbf{r}_{\alpha \beta}} \wedge \mathbf{u}_{\mathbf{r}_{\alpha \gamma} \mid}\right|} \cdot \frac{\mathbf{u}_{\mathbf{r}_{\alpha \kappa}} \wedge \mathbf{u}_{\mathbf{r}_{\alpha \lambda}}}{\left|\mathbf{u}_{\mathbf{r}_{\alpha \kappa}} \wedge \mathbf{u}_{\mathbf{r}_{\alpha \lambda} \mid}\right|} \\
& =\frac{\mathbf{u}_{\mathbf{r}_{\alpha \beta}} \cdot \mathbf{u}_{\mathbf{r}_{\alpha \kappa}} \mathbf{u}_{\mathbf{r}_{\alpha \gamma}} \cdot \mathbf{u}_{\mathbf{r}_{\alpha \lambda}}-\mathbf{u}_{\mathbf{r}_{\alpha \gamma}} \cdot \mathbf{u}_{\mathbf{r}_{\alpha \kappa}} \mathbf{u}_{\mathbf{r}_{\alpha \beta}} \cdot \mathbf{u}_{\mathbf{r}_{\alpha \lambda}}}{\sin \theta_{\beta \alpha \gamma} \sin \theta_{\kappa \alpha \lambda}},
\end{aligned}
$$

where the last equality follows from Laplace's expansion rule (A1) and from the definitions (21) and (23). By expressing the inner products in terms of the valence angles, this reads as

$\sin \theta_{\beta \alpha \gamma} \sin \theta_{\kappa \alpha \lambda} \cos \xi_{\alpha \beta \gamma \kappa \lambda}$
$\quad=\cos \theta_{\beta \alpha \kappa} \cos \theta_{\gamma \alpha \lambda}-\cos \theta_{\gamma \alpha \kappa} \cos \theta_{\beta \alpha \lambda}$.

The gradients are obtained by differentiating both sides of Eq. (62). This results in a set of equations of the type,

$$
\begin{aligned}
\cos \theta_{\beta \alpha \gamma}\left(\partial_{\mathbf{x}_{\beta}} \theta_{\beta \alpha \gamma}\right) \sin \theta_{\kappa \alpha \lambda} \cos \xi_{\alpha \beta \gamma \kappa \lambda} \\
-\sin \theta_{\beta \alpha \gamma} \sin \theta_{\kappa \alpha \lambda} \sin \xi_{\alpha \beta \gamma \kappa \lambda} \partial_{\mathbf{x}_{\beta}} \xi_{\alpha \beta \gamma \kappa \lambda} \\
=-\cos \theta_{\gamma \alpha \lambda} \sin \theta_{\beta \alpha \kappa} \partial_{\mathbf{x}_{\beta}} \theta_{\beta \alpha \kappa} \\
\quad+\cos \theta_{\gamma \alpha \kappa} \sin \theta_{\beta \alpha \lambda} \partial_{\mathbf{x}_{\beta}} \theta_{\beta \alpha \lambda},
\end{aligned}
$$

which, after the insertion of the known derivatives, reads as 
TABLE I. Gradients of the internal coordinates (see text for the definitions of the coordinates).

$$
\begin{aligned}
& \nabla_{\beta} \varphi_{\alpha \beta \gamma \kappa}=\frac{\mathbf{r}_{\alpha \gamma} \times \mathbf{r}_{\alpha \kappa}}{r_{\alpha \beta} r_{\alpha \gamma} r_{\alpha \kappa}}-\varphi_{\alpha \beta \gamma \kappa} \frac{\mathbf{r}_{\alpha \beta}}{r_{\alpha \beta}^{2}} \\
& \boldsymbol{\nabla}_{\gamma} \varphi_{\alpha \beta \gamma \kappa}=\frac{\mathbf{r}_{\alpha \kappa} \times \mathbf{r}_{\alpha \beta}}{r_{\alpha \beta} r_{\alpha \gamma} r_{\alpha \kappa}}-\varphi_{\alpha \beta \gamma \kappa} \frac{\mathbf{r}_{\alpha \gamma}}{r_{\alpha \gamma}^{2}} \\
& \nabla_{\kappa} \varphi_{\alpha \beta \gamma \kappa}=\frac{\mathbf{r}_{\alpha \beta} \times \mathbf{r}_{\alpha \gamma}}{r_{\alpha \beta} r_{\alpha \gamma} r_{\alpha \kappa}}-\varphi_{\alpha \beta \gamma \kappa} \frac{\mathbf{r}_{\alpha \kappa}}{r_{\alpha \kappa}^{2}} \\
& \nabla_{\alpha} \varphi_{\alpha \beta \gamma \kappa}=-\left(\nabla_{\beta} \varphi_{\alpha \beta \gamma \kappa}+\nabla_{\gamma} \varphi_{\alpha \beta \gamma \kappa}+\nabla_{\kappa} \varphi_{\alpha \beta \gamma \kappa}\right) \\
& \boldsymbol{\nabla}_{\beta} \xi_{\alpha \beta \gamma \kappa \lambda}=-\frac{\cos \theta_{\gamma \alpha \kappa}}{\sin \xi_{\alpha \beta \gamma \kappa \lambda} \sin \theta_{\beta \alpha \gamma} \sin \theta_{\kappa \alpha \lambda}}\left(\frac{\mathbf{u}_{\mathbf{r}_{\alpha \beta}} \cos \theta_{\beta \alpha \lambda}-\mathbf{u}_{\mathbf{r}_{\alpha \lambda}}}{r_{\alpha \beta}}\right)+\frac{\cos \theta_{\gamma \alpha \lambda}}{\sin \xi_{\alpha \beta \gamma \kappa \lambda} \sin \theta_{\beta \alpha \gamma} \sin \theta_{\kappa \alpha \lambda}}\left(\frac{\mathbf{u}_{\mathbf{r}_{\alpha \beta}} \cos \theta_{\beta \alpha \kappa}-\mathbf{u}_{\mathbf{r}_{\alpha \kappa}}}{r_{\alpha \beta}}\right)+\frac{\cos \theta_{\beta \alpha \gamma} \cos \xi_{\alpha \beta \gamma \kappa \lambda}}{\sin \xi_{\alpha \beta \gamma \kappa \lambda} \sin ^{2} \theta_{\beta \alpha \gamma}}\left(\frac{\mathbf{u}_{\alpha \beta}}{\cos \theta_{\beta \alpha \gamma}-\mathbf{u}_{\mathbf{r}_{\alpha \gamma}}}\right) \\
& \boldsymbol{\nabla}_{\gamma} \xi_{\alpha \beta \gamma \kappa \lambda}=-\frac{\cos \theta_{\beta \alpha \lambda}}{\sin \xi_{\alpha \beta \gamma \kappa \lambda} \sin \theta_{\beta \alpha \gamma} \sin \theta_{\kappa \alpha \lambda}}\left(\frac{\mathbf{u}_{\mathbf{r}_{\alpha \gamma}} \cos \theta_{\gamma \alpha \kappa}-\mathbf{u}_{\mathbf{r}_{\alpha \kappa}}}{r_{\alpha \gamma}}\right)+\frac{\cos \theta_{\beta \alpha \kappa}}{\sin \xi_{\alpha \beta \gamma \kappa \lambda} \sin \theta_{\beta \alpha \gamma} \sin \theta_{\kappa \alpha \lambda}}\left(\frac{\mathbf{u}_{\mathbf{r}_{\alpha \gamma}} \cos \theta_{\gamma \alpha \lambda}-\mathbf{u}_{\mathbf{r}_{\alpha \lambda}}}{r_{\alpha \gamma}}\right)+\frac{\cos \theta_{\beta \alpha \gamma} \cos \xi_{\alpha \beta \gamma \kappa \lambda}}{\sin \xi_{\alpha \beta \gamma \kappa \lambda}} \sin ^{2} \theta_{\beta \alpha \gamma}\left(\frac{\mathbf{u}_{\alpha \gamma} \cos \theta_{\beta \alpha \gamma}-\mathbf{u}_{\mathbf{r}_{\alpha \beta}}}{r_{\alpha \gamma}}\right) \\
& \nabla_{\kappa} \xi_{\alpha \beta \gamma \kappa \lambda}=-\frac{\cos \theta_{\beta \alpha \lambda}}{\sin \xi_{\alpha \beta \gamma \kappa \lambda} \sin \theta_{\beta \alpha \gamma} \sin \theta_{\kappa \alpha \lambda}}\left(\frac{\mathbf{u}_{\mathbf{r}_{\alpha \kappa}} \cos \theta_{\gamma \alpha \kappa}-\mathbf{u}_{\mathbf{r}_{\alpha \gamma}}}{r_{\alpha \kappa}}\right)+\frac{\cos \theta_{\gamma \alpha \lambda}}{\sin \xi_{\alpha \beta \gamma \kappa \lambda} \sin \theta_{\beta \alpha \gamma} \sin \theta_{\kappa \alpha \lambda}}\left(\frac{\mathbf{u}_{\mathbf{r}_{\alpha \kappa}} \cos \theta_{\beta \alpha \kappa}-\mathbf{u}_{\mathbf{r}_{\alpha \beta}}}{r_{\alpha \kappa}}\right)+\frac{\cos \theta_{\kappa \alpha \lambda} \cos \xi_{\alpha \beta \gamma \kappa \lambda}}{\sin \xi_{\alpha \beta \gamma \kappa \lambda}} \sin ^{2} \theta_{\kappa \alpha \lambda}\left(\frac{\mathbf{u}_{\alpha \kappa} \cos \theta_{\kappa \alpha \lambda}-\mathbf{u}_{\mathbf{r}_{\alpha \lambda}}}{r_{\alpha \kappa}}\right) \\
& \nabla_{\lambda} \xi_{\alpha \beta \gamma \kappa \lambda}=-\frac{\cos \theta_{\gamma \alpha \kappa}}{\sin \xi_{\alpha \beta \gamma \kappa \lambda} \sin \theta_{\beta \alpha \gamma} \sin \theta_{\kappa \alpha \lambda}}\left(\frac{\mathbf{u}_{\alpha \lambda} \cos \theta_{\beta \alpha \lambda}-\mathbf{u}_{\mathbf{r}_{\alpha \beta}}}{r_{\alpha \lambda}}\right)+\frac{\cos \theta_{\beta \alpha \kappa}}{\sin \xi_{\alpha \beta \gamma \kappa \lambda} \sin \theta_{\beta \alpha \gamma} \sin \theta_{\kappa \alpha \lambda}}\left(\frac{\mathbf{u}_{\mathbf{r}_{\alpha \lambda}} \cos \theta_{\gamma \alpha \lambda}-\mathbf{u}_{\mathbf{r}_{\alpha \gamma}}}{r_{\alpha \lambda}}\right)+\frac{\cos \theta_{\kappa \alpha \lambda} \cos \xi_{\alpha \beta \gamma \kappa \lambda}}{\sin \xi_{\alpha \beta \gamma \kappa \lambda} \sin \theta^{2} \theta_{\kappa \alpha \lambda}}\left(\frac{\mathbf{u}_{\alpha \lambda} \cos \theta_{\kappa \alpha \lambda}-\mathbf{u}_{\mathbf{r}_{\alpha \kappa}}}{r_{\alpha \lambda}}\right) \\
& \boldsymbol{\nabla}_{\alpha} \xi_{\alpha \beta \gamma \kappa \lambda}=-\left(\nabla_{\beta} \xi_{\alpha \beta \gamma \kappa \lambda}+\nabla_{\gamma} \xi_{\alpha \beta \gamma \kappa \lambda}+\nabla_{\kappa} \xi_{\alpha \beta \gamma \kappa \lambda}+\nabla_{\lambda} \xi_{\alpha \beta \gamma \kappa \lambda}\right) \\
& \boldsymbol{\nabla}_{\beta} Z_{\alpha \beta \gamma \kappa}=\frac{\mathbf{r}_{\gamma \kappa} \times \mathbf{r}_{\alpha \gamma}}{2 r_{\alpha \beta} r_{\gamma \kappa} \sin \theta_{\alpha \beta \gamma \kappa}}-\left(\frac{\mathbf{u}_{\mathbf{r}_{\alpha \beta}}-\mathbf{u}_{\mathbf{r}_{\gamma \kappa}} \cos \theta_{\alpha \beta \gamma \kappa}}{r_{\alpha \beta} \sin ^{2} \theta_{\alpha \beta \gamma \kappa}}\right) Z_{\alpha \beta \gamma \kappa} \\
& \nabla_{\gamma} Z_{\alpha \beta \gamma \kappa}=\frac{\mathbf{r}_{\alpha \beta} \times \mathbf{r}_{\alpha \kappa}}{2 r_{\alpha \beta} r_{\gamma \kappa} \sin \theta_{\alpha \beta \gamma \kappa}}-\left(\frac{\mathbf{u}_{\mathbf{r}_{\alpha \beta}} \cos \theta_{\alpha \beta \gamma \kappa}-\mathbf{u}_{\mathbf{r}_{\gamma \kappa}}}{r_{\gamma \kappa} \sin ^{2} \theta_{\alpha \beta \gamma \kappa}}\right) Z_{\alpha \beta \gamma \kappa} \\
& \nabla_{\kappa} Z_{\alpha \beta \gamma \kappa}=\frac{\mathbf{r}_{\alpha \gamma} \times \mathbf{r}_{\alpha \beta}}{2 r_{\alpha \beta} r_{\gamma \kappa} \sin \theta_{\alpha \beta \gamma \kappa}}-\left(\frac{\mathbf{u}_{\mathbf{r}_{\gamma \kappa}}-\mathbf{u}_{\mathbf{r}_{\alpha \beta}} \cos \theta_{\alpha \beta \gamma \kappa}}{r_{\gamma \kappa} \sin ^{2} \theta_{\alpha \beta \gamma \kappa}}\right) Z_{\alpha \beta \gamma \kappa} \\
& \nabla_{\alpha} Z_{\alpha \beta \gamma \kappa}=-\left(\nabla_{\beta} Z_{\alpha \beta \gamma \kappa}+\nabla_{\gamma} Z_{\alpha \beta \gamma \kappa}+\nabla_{\kappa} Z_{\alpha \beta \gamma \kappa}\right) \\
& \boldsymbol{\nabla}_{\beta} \varpi_{\alpha \beta \gamma \kappa}=\left(\frac{\cos \varpi_{\alpha \beta \gamma \kappa} \cos \theta_{\beta \alpha \gamma} \sin \theta_{\beta \alpha \kappa}-\cos \theta_{\beta \alpha \kappa} \sin \theta_{\beta \alpha \gamma}}{\sin \varpi_{\alpha \beta \gamma} \sin \theta_{\beta \alpha \gamma} \sin \theta_{\beta \alpha \kappa}}\right)\left(\frac{\mathbf{u}_{\mathbf{r}_{\alpha \beta}} \cos \theta_{\beta \alpha \gamma}-\mathbf{u}_{\mathbf{r}_{\alpha \gamma}}}{r_{\alpha \beta} \sin \theta_{\beta \alpha \gamma}}\right)+\left(\frac{\cos \varpi_{\alpha \beta \gamma \kappa} \sin \theta_{\beta \alpha \gamma} \cos \theta_{\beta \alpha \kappa}-\sin \theta_{\beta \alpha \kappa} \cos \theta_{\beta \alpha \gamma}}{\sin \varpi_{\alpha \beta \gamma \kappa} \sin \theta_{\beta \alpha \gamma} \sin \theta_{\beta \alpha \kappa}}\right)\left(\frac{\mathbf{u}_{\mathbf{r}_{\alpha \beta}} \cos \theta_{\beta \alpha \kappa}-\mathbf{u}_{\mathbf{r}_{\alpha \kappa}}}{r_{\alpha \beta} \sin \theta_{\beta \alpha \kappa}}\right) \\
& \boldsymbol{\nabla}_{\gamma} \varpi_{\alpha \beta \gamma \kappa}=\left(\frac{\sin \theta_{\gamma \alpha \kappa}}{\sin \varpi_{\alpha \beta \gamma \kappa} \sin \theta_{\beta \alpha \gamma} \sin \theta_{\beta \alpha \kappa}}\right)\left(\frac{\mathbf{u}_{\mathbf{r}_{\alpha \gamma}} \cos \theta_{\gamma \alpha \kappa}-\mathbf{u}_{\mathbf{r}_{\alpha \kappa}}}{r_{\alpha \gamma} \sin \theta_{\gamma \alpha \kappa}}\right)+\left(\frac{\cos \varpi_{\alpha \beta \gamma \kappa} \cos \theta_{\beta \alpha \gamma} \sin \theta_{\beta \alpha \kappa}-\cos \theta_{\beta \alpha \kappa} \sin \theta_{\beta \alpha \gamma}}{\sin \varpi_{\alpha \beta \gamma \kappa} \sin \theta_{\beta \alpha \gamma} \sin \theta_{\beta \alpha \kappa}}\right)\left(\frac{\mathbf{u}_{\mathbf{r}_{\alpha \gamma}} \cos \theta_{\beta \alpha \gamma}-\mathbf{u}_{\mathbf{r}_{\alpha \beta}}}{r_{\alpha \gamma} \sin \theta_{\beta \alpha \gamma}}\right) \\
& \nabla_{\kappa} \varpi_{\alpha \beta \gamma \kappa}=\left(\frac{\sin \theta_{\gamma \alpha \kappa}}{\sin \varpi_{\alpha \beta \gamma \kappa} \sin \theta_{\beta \alpha \gamma} \sin \theta_{\beta \alpha \kappa}}\right)\left(\frac{\mathbf{u}_{\mathbf{r}_{\alpha \kappa}} \cos \theta_{\gamma \alpha \kappa}-\mathbf{u}_{\mathbf{r}_{\alpha \gamma}}}{r_{\alpha \kappa} \sin \theta_{\gamma \alpha \kappa}}\right)+\left(\frac{\cos \varpi_{\alpha \beta \gamma \kappa} \cos \theta_{\beta \alpha \kappa} \sin \theta_{\beta \alpha \gamma}-\cos \theta_{\beta \alpha \gamma} \sin \theta_{\beta \alpha \kappa}}{\sin \varpi_{\alpha \beta \gamma \kappa} \sin \theta_{\beta \alpha \gamma} \sin \theta_{\beta \alpha \kappa}}\right)\left(\frac{\mathbf{u}_{\mathbf{r}_{\alpha \kappa}} \cos \theta_{\beta \alpha \kappa}-\mathbf{u}_{\mathbf{r}_{\alpha \beta}}}{r_{\alpha \kappa} \sin \theta_{\beta \alpha \kappa}}\right) \\
& \nabla_{\alpha} \varpi_{\alpha \beta \gamma \kappa}=-\left(\nabla_{\beta} \varpi_{\alpha \beta \gamma \kappa}+\nabla_{\gamma} \varpi_{\alpha \beta \gamma \kappa}+\nabla_{\kappa} \varpi_{\alpha \beta \gamma \kappa}\right)
\end{aligned}
$$

$$
\begin{aligned}
\partial_{\mathbf{x}_{\beta}} \xi_{\alpha \beta \gamma \kappa \lambda}= & -\frac{\cos \theta_{\gamma \alpha \kappa}}{\sin \xi_{\alpha \beta \gamma \kappa \lambda} \sin \theta_{\beta \alpha \gamma} \sin \theta_{\kappa \alpha \lambda}}\left(\frac{\mathbf{u}_{\alpha \beta} \cos \theta_{\beta \alpha \lambda}-\mathbf{u}_{\mathbf{r}_{\alpha \lambda}}}{r_{\alpha \beta}}\right)+\frac{\cos \theta_{\gamma \alpha \lambda}}{\sin \xi_{\alpha \beta \gamma \kappa \lambda} \sin \theta_{\beta \alpha \gamma} \sin \theta_{\kappa \alpha \lambda}}\left(\frac{\mathbf{u}_{\alpha \beta} \cos \theta_{\beta \alpha \kappa}-\mathbf{u}_{\mathbf{r}_{\alpha \kappa}}}{r_{\alpha \beta}}\right) \\
& +\frac{\cos \theta_{\beta \alpha \gamma} \cos \xi_{\alpha \beta \gamma \kappa \lambda}}{\sin \xi_{\alpha \beta \gamma \kappa \lambda} \sin ^{2} \theta_{\beta \alpha \gamma}}\left(\frac{\mathbf{u}_{\mathbf{r}_{\alpha \beta}} \cos \theta_{\beta \alpha \gamma}-\mathbf{u}_{\mathbf{r}_{\alpha \gamma}}}{r_{\alpha \beta}}\right) .
\end{aligned}
$$

Similarly,

$$
\begin{aligned}
\partial_{\mathbf{x}_{\gamma} \xi_{\alpha \beta \gamma \kappa \lambda}=} & -\frac{\cos \theta_{\beta \alpha \lambda}}{\sin \xi_{\alpha \beta \gamma \kappa \lambda} \sin \theta_{\beta \alpha \gamma} \sin \theta_{\kappa \alpha \lambda}}\left(\frac{\mathbf{u}_{\mathbf{r}_{\alpha \gamma}} \cos \theta_{\gamma \alpha \kappa}-\mathbf{u}_{\mathbf{r}_{\alpha \kappa}}}{r_{\alpha \gamma}}\right)+\frac{\cos \theta_{\beta \alpha \kappa}}{\sin \xi_{\alpha \beta \gamma \kappa \lambda} \sin \theta_{\beta \alpha \gamma} \sin \theta_{\kappa \alpha \lambda}}\left(\frac{\mathbf{u}_{\alpha \gamma} \cos \theta_{\gamma \alpha \lambda}-\mathbf{u}_{\mathbf{r}_{\alpha \lambda}}}{r_{\alpha \gamma}}\right) \\
& +\frac{\cos \theta_{\beta \alpha \gamma} \cos \xi_{\alpha \beta \gamma \kappa \lambda}}{\sin \xi_{\alpha \beta \gamma \kappa \lambda} \sin ^{2} \theta_{\beta \alpha \gamma}}\left(\frac{\mathbf{u}_{\mathbf{r}_{\alpha \gamma}} \cos \theta_{\beta \alpha \gamma}-\mathbf{u}_{\mathbf{r}_{\alpha \beta}}}{r_{\alpha \gamma}}\right) .
\end{aligned}
$$


The gradients $\partial_{\mathbf{x}_{\kappa}} \xi_{\alpha \beta \gamma \kappa \lambda}$ and $\partial_{\mathbf{x}_{\lambda}} \xi_{\alpha \beta \gamma \kappa \lambda}$ are given by exchanging the indices $\beta$ and $\kappa$ and the indices $\gamma$ and $\lambda$ in Eqs. (64) and (65). They are tabulated in Table I. The central atom gradient is given by

$$
\begin{aligned}
\partial_{\mathbf{x}_{\alpha}} \xi_{\alpha \beta \gamma \kappa \lambda}= & -\left(\partial_{\mathbf{x}_{\beta}} \xi_{\alpha \beta \gamma \kappa \lambda}+\partial_{\mathbf{x}_{\gamma}} \xi_{\alpha \beta \gamma \kappa \lambda}+\partial_{\mathbf{x}_{\kappa}} \xi_{\alpha \beta \gamma \kappa \lambda}\right. \\
& \left.+\partial_{\mathbf{x}_{\lambda}} \xi_{\alpha \beta \gamma \kappa \lambda}\right) .
\end{aligned}
$$

\section{B. Some ring coordinates}

Ring coordinates are especially designed to represent the relative motion of different parts of the ring (such as the ring puckering) or the motion of the ring as a whole (such as the ring breathing coordinate) ${ }^{25}$ In the latter case, the ring coordinates are typically symmetrized linear combinations of some already familiar internal coordinates (for example, the breathing coordinate is a sum of ring bond lengths). An important property for any ring structure is the ring closure; the sum of all the ring bond vectors is zero,

$$
\sum \mathbf{r}_{\alpha \beta}=0 \text {. }
$$

Generally, if there are $R$ possible cyclic paths, then there exist $R$ ring closure conditions (see Fig. 5). Luckily, these closure conditions do not complicate the gradient calculations. The reason is obvious; any independent or dependent relative position vector $\mathbf{r}_{\alpha \beta}=\mathbf{x}_{\beta}-\mathbf{x}_{\alpha}$ is a function of end atom position vectors only. Hence, the closure conditions Eq. (67) can be ignored in the gradient calculations [but if need be, they can be substituted to the gradient $\boldsymbol{\nabla}_{\alpha} q_{i}\left(\mathbf{r}_{1}, \mathbf{r}_{2}, \ldots, \mathbf{r}_{N+R}\right)$, which produces the same result $\boldsymbol{\nabla}_{\alpha} q_{i}\left(\mathbf{r}_{1}, \mathbf{r}_{2}, \ldots, \mathbf{r}_{N}\right)$ as if the ring coordinate $q_{i}$ had been expressed in the first place as a function of independent ring vectors $\left.\mathbf{r}_{1}, \mathbf{r}_{2}, \ldots, \mathbf{r}_{N}\right]$. However, the closure conditions can be expressed in terms of coordinates, and hence used to eliminate the dependent coordinates by the method given in the Ref. 16. Usually, the inner products $\left(\boldsymbol{\nabla}_{\alpha} q_{i}\right) \cdot\left(\nabla_{\alpha} q_{j}\right)$ of the ring coordinates are more easily expressed in terms of some independent plus dependent coordinates, than in terms of independent coordinates only.

By using geometric algebra, the gradients for any ring coordinate are easily calculated. In the next subsections, the gradients are calculated for the first time for the familiar ring breathing coordinate and the ring puckering coordinate. Furthermore, I define a diagonal book angle coordinate to describe the relative orientation of two parts of the ring, and derive its gradients.

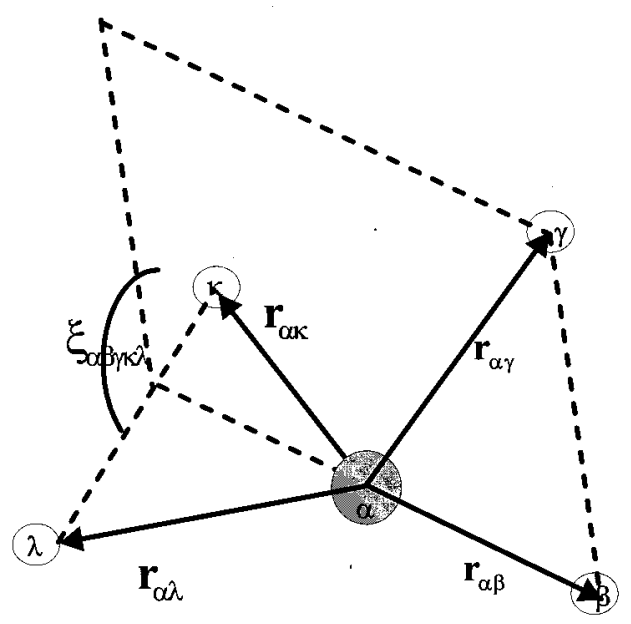

FIG. 4. Twisting $\xi_{\alpha \beta \gamma \kappa \lambda}$.

\section{Ring breathing}

In the ring breathing mode, the length of the ring changes in a symmetric fashion (this is depicted for a fouratom ring in Fig. 6). A natural definition of the ring breathing coordinate for an $\mathrm{N}$-atom ring is therefore

$$
\epsilon_{123 \cdots N}=r_{12}+r_{23}+\cdots+r_{N-1, N}+r_{N, 1} .
$$

Using the bond vectors, this can be written as

$$
\begin{aligned}
\epsilon_{123 \cdots N}= & \left(\mathbf{r}_{12} \cdot \mathbf{r}_{12}\right)^{1 / 2}+\left(\mathbf{r}_{23} \cdot \mathbf{r}_{23}\right)^{1 / 2}+\cdots \\
& +\left(\mathbf{r}_{N-1, N} \cdot \mathbf{r}_{N-1, N}\right)^{1 / 2}+\left(\mathbf{r}_{N, 1} \cdot \mathbf{r}_{N, 1}\right)^{1 / 2} .
\end{aligned}
$$

By regarding all the bond vectors as independent vectors, there are gradients of the type,

$$
\partial_{\mathbf{x}_{\alpha}} \epsilon_{123 \cdots N}=\mathbf{u}_{\mathbf{r}_{\alpha-1, \alpha}}-\mathbf{u}_{\mathbf{r}_{\alpha, \alpha+1}} .
$$

For example,

$$
\partial_{\mathbf{x}_{2}} \epsilon_{123 \cdots N}=\mathbf{u}_{\mathbf{r}_{1,2}}-\mathbf{u}_{\mathbf{r}_{2,3}} .
$$

Due to the ring closure condition, one of the ring vectors, say $\mathbf{r}_{N, 1}$, is equal to minus of the sum of the other ring vectors,

$$
\mathbf{r}_{N, 1}=-\left(\mathbf{r}_{12}+\mathbf{r}_{23}+\cdots+\mathbf{r}_{N-1, N}\right) \text {. }
$$

If wanted (although this is unnecessary), the dependent ring vector can be written in terms of independent ring vectors, and hence the gradients $\partial_{\mathbf{x}_{N}} \epsilon_{123 \cdots N}$ and $\partial_{\mathbf{x}_{1}} \epsilon_{123 \cdots N}$ become

$$
\partial_{\mathbf{x}_{N}} \epsilon_{123 \cdots N}=\mathbf{u}_{\mathbf{r}_{N-1, N}}-\frac{\mathbf{r}_{N, 1}}{r_{N, 1}}=\mathbf{u}_{\mathbf{r}_{N-1, N}}+\frac{\mathbf{r}_{12}+\mathbf{r}_{23}+\cdots+\mathbf{r}_{N-1, N}}{\left[r_{12}^{2}+2 \mathbf{r}_{12} \cdot \mathbf{r}_{23}+\cdots+2 \mathbf{r}_{12} \cdot \mathbf{r}_{N-1, N}+r_{23}^{2}+2 \mathbf{r}_{23} \cdot \mathbf{r}_{34}+\cdots+r_{N-1, N}^{2}\right]^{1 / 2}}
$$

and

$$
\partial_{\mathbf{x}_{1}} \epsilon_{123 \cdots N}=\frac{\mathbf{r}_{N, 1}}{r_{N, 1}}-\mathbf{u}_{\mathbf{r}_{12}}=-\frac{\mathbf{r}_{12}+\mathbf{r}_{23}+\cdots+\mathbf{r}_{N-1, N}}{\left[r_{12}^{2}+2 \mathbf{r}_{12} \cdot \mathbf{r}_{23}+\cdots+2 \mathbf{r}_{12} \cdot \mathbf{r}_{N-1, N}+r_{23}^{2}+2 \mathbf{r}_{23} \cdot \mathbf{r}_{34}+\cdots+r_{N-1, N}^{2}\right]^{1 / 2}}-\mathbf{u}_{\mathbf{r}_{12}} .
$$

These results are the same as if the dependent ring vector would have been eliminated and the gradients taken. This would have produced 


$$
\partial_{\mathbf{x}_{\alpha}} \epsilon_{123 \cdots N}=\partial_{\mathbf{x}_{\alpha}}\left(r_{12}+r_{23}+\cdots+r_{N-1, N}\right)+\frac{\partial_{\mathbf{x}_{\alpha}}\left(r_{12}^{2}+2 \mathbf{r}_{12} \cdot \mathbf{r}_{23}+\cdots+2 \mathbf{r}_{12} \cdot \mathbf{r}_{N-1, N}+r_{23}^{2}+2 \mathbf{r}_{23} \cdot \mathbf{r}_{34}+\cdots+r_{N-1, N}^{2}\right)}{2\left[r_{12}^{2}+2 \mathbf{r}_{12} \cdot \mathbf{r}_{23}+\cdots+2 \mathbf{r}_{12} \cdot \mathbf{r}_{N-1, N}+r_{23}^{2}+2 \mathbf{r}_{23} \cdot \mathbf{r}_{34}+\cdots+r_{N-1, N}^{2}\right]^{1 / 2}}
$$

With the help of the vector derivatives given in Appendix A one can confirm that this gives Eq. (70) for $\alpha=2,3, \cdots, N$ -1 , Eq. (74) for $\alpha=1$, and Eq. (73) for $\alpha=N$.

\section{Ring puckering}

The absolute value of the ring puckering coordinate $Z_{\alpha \beta \gamma \kappa}$ is defined $^{25}$ as the half of the distance between the two ring diagonals $\mathbf{r}_{\alpha \beta}=\mathbf{x}_{\beta}-\mathbf{x}_{\alpha}$ and $\mathbf{r}_{\gamma \kappa}=\mathbf{x}_{\kappa}-\mathbf{x}_{\gamma}$, i.e.,

$$
\left|Z_{\alpha \beta \gamma \kappa}\right|=\frac{d_{\alpha \beta \gamma \kappa}}{2},
$$

where $d_{\alpha \beta \gamma \kappa}=\left(\mathbf{d}_{\alpha \beta \gamma \kappa}^{\dagger} \cdot \mathbf{d}_{\alpha \beta \gamma \kappa}\right)^{1 / 2} \geqslant 0$ is the minimum chord distance, and

$$
\begin{aligned}
\mathbf{d}_{\alpha \beta \gamma \kappa} & =\left(\mathbf{x}_{\gamma}-\mathbf{x}_{\alpha}\right) \wedge \mathbf{r}_{\alpha \beta} \wedge \mathbf{r}_{\gamma \kappa}\left(\mathbf{r}_{\alpha \beta} \wedge \mathbf{r}_{\gamma \kappa}\right)^{-1} \\
& =\mathbf{r}_{\alpha \gamma} \wedge \mathbf{r}_{\alpha \beta} \wedge \mathbf{r}_{\gamma \kappa}\left(\mathbf{r}_{\alpha \beta} \wedge \mathbf{r}_{\gamma \kappa}\right)^{-1}
\end{aligned}
$$

is the directance from the line $\mathbf{r}_{\alpha \beta}$ to the line $\mathbf{r}_{\gamma \kappa}$ (see the answer to exercise 6.7 in the Sec. 2-6 of Ref. 19). The ring puckering coordinate $Z_{2413}$ is depicted in the Fig. 7 for a four atom ring. The sign of the puckering coordinate reveals, if the nuclei $\alpha$ and $\beta$ (or $\gamma$ and $\kappa$ ) have moved "above" or "below" the reference plane. Hence, a natural definition for the ring puckering is

$$
Z_{\alpha \beta \gamma \kappa}=-\frac{i \mathbf{r}_{\alpha \gamma} \wedge \mathbf{r}_{\alpha \beta} \wedge \mathbf{r}_{\gamma \kappa}}{2\left|\mathbf{r}_{\alpha \beta} \wedge \mathbf{r}_{\gamma \kappa}\right|}=-\frac{i \mathbf{r}_{\alpha \gamma} \wedge \mathbf{r}_{\alpha \beta} \wedge \mathbf{r}_{\gamma \kappa}}{2 r_{\alpha \beta} r_{\gamma \kappa} \sin \theta_{\alpha \beta \gamma \kappa}},
$$

where $i$ is the unit trivector and $\theta_{\alpha \beta \gamma \kappa}$ stands for the angle between $\mathbf{r}_{\alpha \beta}$ and $\mathbf{r}_{\gamma \kappa}$. In the current notation, the valence angles can be written as $\theta_{\beta \alpha \gamma} \equiv \theta_{\alpha \beta \alpha \gamma}$. If the dextral order of the relative position vectors $\mathbf{r}_{\alpha \gamma}, \mathbf{r}_{\alpha \beta}$, and $\mathbf{r}_{\gamma \kappa}$ is fixed as $\left\{\mathbf{r}_{\alpha \gamma}, \mathbf{r}_{\alpha \beta}, \mathbf{r}_{\gamma \kappa}\right\}$, the positive sign of $Z_{\alpha \beta \gamma \kappa}$ means that the relative position vectors $\mathbf{r}_{\alpha \gamma}, \mathbf{r}_{\alpha \beta}$, and $\mathbf{r}_{\gamma \kappa}$ form a righthanded set (the diagonal $\mathbf{r}_{\gamma \kappa}$ points "upward"). Likewise, if the sign of $Z_{\alpha \beta \gamma \kappa}$ is negative, the relative position vectors form a left-handed set (the diagonal $\mathbf{r}_{\gamma \kappa}$ points "downward'"). Note, that in Fig. 7 the triplet $\left\{\mathbf{r}_{21}, \mathbf{r}_{24}, \mathbf{r}_{13}\right\}$ is right- handed (and the diagonal $\mathbf{r}_{13}$ points "upward"), so the value of the corresponding ring puckering coordinate $Z_{2413}$ is positive.

The gradient $\partial_{\mathbf{x}_{\beta}} Z_{\alpha \beta \gamma \kappa}$ is obtained by taking the vector derivative of both sides of Eq. (78). This produces

$$
\begin{aligned}
& \partial_{\mathbf{x}_{\beta}} Z_{\alpha \beta \gamma \kappa}=-\frac{i \partial_{\mathbf{x}_{\beta}}\left(\mathbf{r}_{\alpha \gamma} \wedge \mathbf{r}_{\alpha \beta} \wedge \mathbf{r}_{\gamma \kappa}\right)}{2 r_{\alpha \beta} r_{\gamma \kappa} \sin \theta_{\alpha \beta \gamma \kappa}} \\
& +\frac{i\left(\partial_{\mathbf{x}_{\beta}} r_{\alpha \beta}\right)\left(\mathbf{r}_{\alpha \gamma} \wedge \mathbf{r}_{\alpha \beta} \wedge \mathbf{r}_{\gamma \kappa}\right)}{2 r_{\alpha \beta}^{2} r_{\gamma \kappa} \sin \theta_{\alpha \beta \gamma \kappa}} \\
& +\frac{i \cos \theta_{\alpha \beta \gamma \kappa}\left(\partial_{\mathbf{x}_{\beta}} \theta_{\alpha \beta \gamma \kappa}\right) \mathbf{r}_{\alpha \gamma} \wedge \mathbf{r}_{\alpha \beta} \wedge \mathbf{r}_{\gamma \kappa}}{2 r_{\alpha \beta} r_{\gamma \kappa} \sin ^{2} \theta_{\alpha \beta \gamma \kappa}},
\end{aligned}
$$

where the gradients of the angles $\theta_{\alpha \beta \gamma \kappa}$ are given by simple vectorial differentiation (like in the case of the valence angles) as

$$
\begin{gathered}
\partial_{\mathbf{x}_{\beta}} \theta_{\alpha \beta \gamma \kappa}=\frac{\mathbf{u}_{\mathbf{r}_{\alpha \beta}} \cos \theta_{\alpha \beta \gamma \kappa}-\mathbf{u}_{\mathbf{r}_{\gamma \kappa}}}{r_{\alpha \beta} \sin \theta_{\alpha \beta \gamma \kappa}}=-\partial_{\mathbf{x}_{\alpha}} \theta_{\alpha \beta \gamma \kappa}, \\
\partial_{\mathbf{x}_{\gamma}} \theta_{\alpha \beta \gamma \kappa}=\frac{\mathbf{u}_{\mathbf{r}_{\alpha \beta}}-\mathbf{u}_{\mathbf{r}_{\gamma \kappa}} \cos \theta_{\alpha \beta \gamma \kappa}}{r_{\gamma \kappa} \sin \theta_{\alpha \beta \gamma \kappa}}=-\partial_{\mathbf{x}_{\kappa}} \theta_{\alpha \beta \gamma \kappa},
\end{gathered}
$$

where $\alpha \neq \beta, \gamma, \kappa, \beta \neq \gamma, \kappa$ and $\gamma \neq \kappa$. The vector derivative of the trivector $\mathbf{r}_{\alpha \gamma} \wedge \mathbf{r}_{\alpha \beta} \wedge \mathbf{r}_{\gamma \kappa}$ is given by

$$
\partial_{\mathbf{x}_{\beta}}\left(\mathbf{r}_{\alpha \gamma} \wedge \mathbf{r}_{\alpha \beta} \wedge \mathbf{r}_{\gamma \kappa}\right)=-\mathbf{r}_{\alpha \gamma} \wedge \mathbf{r}_{\gamma \kappa}=-i \mathbf{r}_{\alpha \gamma} \times \mathbf{r}_{\gamma \kappa}
$$

(see Appendix A). Hence

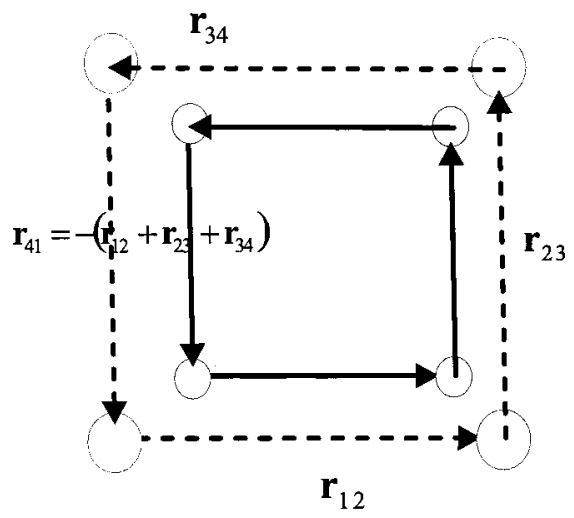

FIG. 6. Ring breathing for a four atom ring. 
$\partial_{\mathbf{x}_{\beta}} Z_{\alpha \beta \gamma \kappa}$

$$
\begin{aligned}
= & \frac{\mathbf{r}_{\gamma \kappa} \times \mathbf{r}_{\alpha \gamma}}{2 r_{\alpha \beta} r_{\gamma \kappa} \sin \theta_{\alpha \beta \gamma \kappa}}+\frac{i \mathbf{u}_{\alpha \beta} \mathbf{r}_{\alpha \gamma} \wedge \mathbf{r}_{\alpha \beta} \wedge \mathbf{r}_{\gamma \kappa}}{2 r_{\alpha \beta}^{2} r_{\gamma \kappa} \sin \theta_{\alpha \beta \gamma \kappa}} \\
& +i\left(\frac{\mathbf{u}_{\alpha \beta} \cos \theta_{\alpha \beta \gamma \kappa}-\mathbf{u}_{\mathbf{r}_{\gamma \kappa}}}{r_{\alpha \beta} \sin \theta_{\alpha \beta \gamma \kappa}}\right) \frac{\cos \theta_{\alpha \beta \gamma \kappa} \mathbf{r}_{\alpha \gamma} \wedge \mathbf{r}_{\alpha \beta} \wedge \mathbf{r}_{\gamma \kappa}}{2 r_{\alpha \beta} r_{\gamma \kappa} \sin ^{2} \theta_{\alpha \beta \gamma \kappa}} \\
= & \frac{\mathbf{r}_{\gamma \kappa} \times \mathbf{r}_{\alpha \gamma}}{2 r_{\alpha \beta} r_{\gamma \kappa} \sin \theta_{\alpha \beta \gamma \kappa}} \\
& -\left(\frac{\mathbf{u}_{\mathbf{r}_{\alpha \beta}}-\mathbf{u}_{\mathbf{r}_{\gamma \kappa}} \cos \theta_{\alpha \beta \gamma \kappa}}{r_{\alpha \beta} \sin ^{2} \theta_{\alpha \beta \gamma \kappa}}\right) Z_{\alpha \beta \gamma \kappa} .
\end{aligned}
$$

Similarly,

$$
\begin{aligned}
& \partial_{\mathbf{x}_{\kappa}} Z_{\alpha \beta \gamma \kappa} \\
& \quad=\frac{\mathbf{r}_{\alpha \gamma} \times \mathbf{r}_{\alpha \beta}}{2 r_{\alpha \beta} r_{\gamma \kappa} \sin \theta_{\alpha \beta \gamma \kappa}}-\left(\frac{\mathbf{u}_{\mathbf{r}_{\gamma \kappa}}-\mathbf{u}_{\mathbf{r}_{\alpha \beta}} \cos \theta_{\alpha \beta \gamma \kappa}}{r_{\gamma \kappa} \sin ^{2} \theta_{\alpha \beta \gamma \kappa}}\right) Z_{\alpha \beta \gamma \kappa} .
\end{aligned}
$$

The other gradients are given in Table I.

\section{Diagonal book angle}

A possible alternative for the ring puckering coordinate is the diagonal book angle $\varpi_{\alpha \beta \gamma \kappa}$ defined as the dihedral angle of the planes spanned by the diagonal $\mathbf{r}_{\alpha \beta}=\mathbf{x}_{\beta}-\mathbf{x}_{\alpha}$ and the bond vectors $\mathbf{r}_{\alpha \gamma}=\mathbf{x}_{\gamma}-\mathbf{x}_{\alpha}$ and $\mathbf{r}_{\alpha \kappa}=\mathbf{x}_{\kappa}-\mathbf{x}_{\alpha}$ (see Fig. 8), i.e.,

$$
\begin{aligned}
\cos \varpi_{\alpha \beta \gamma \kappa} & =\frac{\left(\mathbf{u}_{\mathbf{r}_{\alpha \beta}} \wedge \mathbf{u}_{\mathbf{r}_{\alpha \gamma}}\right)^{\dagger}}{\left|\mathbf{u}_{\mathbf{r}_{\alpha \beta}} \wedge \mathbf{u}_{\mathbf{r}_{\alpha \gamma}}\right|} \cdot \frac{\mathbf{u}_{\mathbf{r}_{\alpha \beta}} \wedge \mathbf{u}_{\mathbf{r}_{\alpha \kappa}}}{\mid \mathbf{u}_{\mathbf{r}_{\alpha \beta}} \wedge \mathbf{u}_{\mathbf{r}_{\alpha \kappa} \mid}} \\
& =\frac{\mathbf{u}_{\mathbf{r}_{\alpha \gamma}} \wedge \mathbf{u}_{\mathbf{r}_{\alpha \beta}}}{\left|\mathbf{u}_{\mathbf{r}_{\alpha \beta}} \wedge \mathbf{u}_{\mathbf{r}_{\alpha \gamma} \mid}\right|} \cdot \frac{\mathbf{u}_{\mathbf{r}_{\alpha \beta}} \wedge \mathbf{u}_{\mathbf{r}_{\alpha \kappa}}}{\mid \mathbf{u}_{\mathbf{r}_{\alpha \beta}} \wedge \mathbf{u}_{\mathbf{r}_{\alpha \kappa} \mid}} \\
& =\frac{\cos \theta_{\gamma \alpha \kappa}-\cos \theta_{\beta \alpha \kappa} \cos \theta_{\beta \alpha \gamma}}{\sin \theta_{\beta \alpha \gamma} \sin \theta_{\beta \alpha \kappa}} .
\end{aligned}
$$

By taking the vector derivative $\partial_{\mathbf{x}_{\beta}}$ of the both sides of Eq. $(85)$,

$$
\begin{aligned}
-\sin \varpi_{\alpha \beta \gamma \kappa} \partial_{\mathbf{x}_{\beta}} \varpi_{\alpha \beta \gamma \kappa}= & \frac{\sin \theta_{\beta \alpha \kappa} \cos \theta_{\beta \alpha \gamma} \partial_{\mathbf{x}_{\beta}} \theta_{\beta \alpha \kappa}+\cos \theta_{\beta \alpha \kappa} \sin \theta_{\beta \alpha \gamma} \partial_{\mathbf{x}_{\beta}} \theta_{\beta \alpha \gamma}}{\sin \theta_{\beta \alpha \gamma} \sin \theta_{\beta \alpha \kappa}} \\
& -\frac{\left(\cos \theta_{\gamma \alpha \kappa}-\cos \theta_{\beta \alpha \kappa} \cos \theta_{\beta \alpha \gamma}\right)\left(\cos \theta_{\beta \alpha \gamma} \sin \theta_{\beta \alpha \kappa} \partial_{\mathbf{x}_{\beta}} \theta_{\beta \alpha \gamma}+\sin \theta_{\beta \alpha \gamma} \cos \theta_{\beta \alpha \kappa} \partial_{\mathbf{x}_{\beta}} \theta_{\beta \alpha \kappa}\right)}{\sin ^{2} \theta_{\beta \alpha \gamma} \sin ^{2} \theta_{\beta \alpha \kappa}}
\end{aligned}
$$

follows, and hence

$$
\begin{aligned}
\partial_{\mathbf{x}_{\beta}} \varpi_{\alpha \beta \gamma \kappa}= & \left(\frac{\cos \varpi_{\alpha \beta \gamma \kappa} \cos \theta_{\beta \alpha \gamma} \sin \theta_{\beta \alpha \kappa}-\cos \theta_{\beta \alpha \kappa} \sin \theta_{\beta \alpha \gamma}}{\sin \varpi_{\alpha \beta \gamma \kappa} \sin \theta_{\beta \alpha \gamma} \sin \theta_{\beta \alpha \kappa}}\right)\left(\frac{\mathbf{u}_{\mathbf{r}_{\alpha \beta}} \cos \theta_{\beta \alpha \gamma}-\mathbf{u}_{\mathbf{r}_{\alpha \gamma}}}{r_{\alpha \beta} \sin \theta_{\beta \alpha \gamma}}\right) \\
& +\left(\frac{\cos \varpi_{\alpha \beta \gamma \kappa} \sin \theta_{\beta \alpha \gamma} \cos \theta_{\beta \alpha \kappa}-\sin \theta_{\beta \alpha \kappa} \cos \theta_{\beta \alpha \gamma}}{\sin \varpi_{\alpha \beta \gamma \kappa} \sin \theta_{\beta \alpha \gamma} \sin \theta_{\beta \alpha \kappa}}\right)\left(\frac{\mathbf{u}_{\mathbf{r}_{\alpha \beta}} \cos \theta_{\beta \alpha \kappa}-\mathbf{u}_{\mathbf{r}_{\alpha \kappa}}}{r_{\alpha \beta} \sin \theta_{\beta \alpha \kappa}}\right) .
\end{aligned}
$$

Similarly,

$$
\begin{aligned}
& \partial_{\mathbf{x}_{\gamma}} \varpi_{\alpha \beta \gamma \kappa} \\
&=\left(\frac{\sin \theta_{\gamma \alpha \kappa}}{\sin \varpi_{\alpha \beta \gamma \kappa} \sin \theta_{\beta \alpha \gamma} \sin \theta_{\beta \alpha \kappa}}\right)\left(\frac{\mathbf{u}_{\mathbf{r}_{\alpha \gamma}} \cos \theta_{\gamma \alpha \kappa}-\mathbf{u}_{\mathbf{r}_{\alpha \kappa}}}{r_{\alpha \gamma} \sin \theta_{\gamma \alpha \kappa}}\right) \\
&+\left(\frac{\cos \varpi_{\alpha \beta \gamma \kappa} \cos \theta_{\beta \alpha \gamma} \sin \theta_{\beta \alpha \kappa}-\cos \theta_{\beta \alpha \kappa} \sin \theta_{\beta \alpha \gamma}}{\sin \varpi_{\alpha \beta \gamma \kappa} \sin \theta_{\beta \alpha \gamma} \sin \theta_{\beta \alpha \kappa}}\right) \\
& \times\left(\frac{\mathbf{u}_{\alpha \gamma} \cos \theta_{\beta \alpha \gamma}-\mathbf{u}_{\mathbf{r}_{\alpha \beta}}}{r_{\alpha \gamma} \sin \theta_{\beta \alpha \gamma}}\right) .
\end{aligned}
$$

The gradient $\partial_{\mathbf{x}_{\kappa}} \varpi_{\alpha \beta \gamma \kappa}$ is obtained from Eq. (88) by exchanging the indices $\gamma$ and $\kappa$. As usual, the central atom gradient is given by

$$
\partial_{\mathbf{x}_{\alpha}} \varpi_{\alpha \beta \gamma \kappa}=-\left(\partial_{\mathbf{x}_{\beta}} \varpi_{\alpha \beta \gamma \kappa}+\partial_{\mathbf{x}_{\gamma}} \varpi_{\alpha \beta \gamma \kappa}+\partial_{\mathbf{x}_{\kappa}} \varpi_{\alpha \beta \gamma \kappa}\right) .
$$

\section{CONCLUSION}

The geometric algebra approach offers some advantages over other methods presented in the literature. First of all, in the geometric algebra approach, the atomic position vectors themselves are manipulated instead of their components, and hence the expressions are simple at each stage of the derivation. This is not the case when Cartesian components and back substitution are used to obtain the vibrational kinetic energy operator. ${ }^{26}$ Furthermore, while the geometric algebra approach is general (that is, independent of any particular molecular part in question), the use of Cartesian components forces one to select an external coordinate frame separately for each molecular part.

The geometric algebra method is also purely algebraic, unlike the conventional "Wilson's $s$-vector method,", 1,2,27 where the gradients $\nabla_{\alpha} q_{i}$ are deduced geometrically at some point by displacing the nucleus $\alpha$ infinitesimally (not by a unit displacement, as is often erroneously stated) and keep- 
ing the others fixed. This gives only the value $\boldsymbol{\nabla}_{\alpha} q_{i}\left(q_{1}^{(e)}, q_{2}^{(e)}, \ldots\right)$ of the gradient in terms of a reference configuration $q_{1}^{(e)}, q_{2}^{(e)}, \ldots$ used (see Appendix B), and one simply assumes that the gradient is obtained from this by replacing $q_{i}^{(e)}$ by $q_{i}$. While in some cases this works, in other cases, where some coordinates are zero at the reference configuration, it may miss some part of the gradient. For example, this would happen if one calculated gradients for out-of-plane bending using a planar reference configuration (this is shown at the end of Appendix B).

Incidentally, by using geometric algebra, Wilson's method could be generalized as ${ }^{20,21}$

$$
\partial_{\mathbf{x}_{\alpha}} q_{i}\left(\mathbf{x}_{\alpha}\right) \equiv \partial_{\mathbf{a}_{\alpha}} q_{i}\left(\mathbf{x}_{\alpha} ; \mathbf{a}_{\alpha}\right),
$$

where

$$
\underline{q_{i}}\left(\mathbf{x}_{\alpha} ; \mathbf{a}_{\alpha}\right)=\lim _{\delta \rightarrow 0} \frac{q_{i}\left(\mathbf{x}_{\alpha}+\delta \mathbf{a}_{\alpha}\right)-q_{i}\left(\mathbf{x}_{\alpha}\right)}{\delta}
$$

is the differential of $q_{i}$ at the arbitrary positions $\mathbf{a}_{\alpha}$ and $\mathbf{x}_{\alpha}$ of nucleus $\alpha$. This is an exact result (i.e., the vector $\mathbf{a}_{\alpha}$ need be neither infinitesimal nor collinear with $\left.\left.\boldsymbol{\nabla}_{\alpha} q_{i}\right|_{\mathbf{x}_{\alpha}}\right)$. However, since one can directly take the gradients of any coordinate, there is no point in calculating them via differentials. In my opinion, "Wilson's $s$-vector method"' is not a practical way to obtain the gradients of the internal coordinates except in the simplest cases, like bond stretchings and valence angle bends, where the correct gradient can be determined geometrically without the need to use Eq. (90).

In the case of ring coordinates, the geometric algebra approach offers an ideal tool in obtaining $g^{\left(q_{i} q_{j}\right)}$ as a sum of the inner product of gradients of the coordinates. On the other hand, the covariant approach of the Ref. 25 is restricted to numerical work. In that approach, one forms the covariant metric tensor $g_{q_{i} q_{j}}=\Sigma_{\alpha} m_{\alpha}\left(d \mathbf{x}_{\alpha} / d q_{i}\right) \cdot\left(d \mathbf{x}_{\alpha} / d q_{j}\right)$, which must be inverted to obtain the reciprocal metric tensor $g^{\left(q_{i} q_{j}\right)}$ that appears in the kinetic energy operator. In practice, this cannot be done analytically, since the elements of $g_{q_{i} q_{j}}$ are functions of the internal coordinates.

It must be added that all the computational complications of the conventional methods are actually due to the imperfections of the Gibbs-Heaviside vectorial system. ${ }^{23}$ For example, since the vectorial division (or a genuine vectorial product for that matter) is not defined in the GibbsHeaviside system, one is forced to use the components of the vectors instead of the vectors themselves in the actual manipulations.

\section{ACKNOWLEDGMENTS}

I wish to thank both The Academy of Finland and The European Commission (FMRX-CT96-0088, TMR network) for financial support. I am also grateful to Professor L. Halonen for useful discussions and valuable comments on the manuscript.

\section{APPENDIX A: SOME USEFUL VECTOR DERIVATIVES AND EXPANSION RULES}

Let $\mathbf{r}_{\alpha \beta}=\mathbf{x}_{\beta}-\mathbf{x}_{\alpha}$ and $\mathbf{r}_{\alpha \gamma}=\mathbf{x}_{\gamma}-\mathbf{x}_{\alpha}$. Furthermore, let $\mathbf{a}$ be a vector and $A_{p}=\mathbf{a}_{1} \wedge \mathbf{a}_{2} \wedge \cdots \wedge \mathbf{a}_{p}$ a $p$-blade independent of $\mathbf{x}_{\alpha}, \mathbf{x}_{\beta}$, and $\mathbf{x}_{\gamma}$. Then, ${ }^{20}$

$\begin{array}{lll}f & \partial_{\mathbf{x}_{\beta} f} & \partial_{\mathbf{x}_{\alpha} f} f \\ \mathbf{r}_{\alpha \beta} & 3 & -3 \\ \mathbf{r}_{\alpha \beta} \mathbf{a} & 3 \mathbf{a} & -3 \mathbf{a} \\ \mathbf{a r}_{\alpha \beta} & -\mathbf{a} & \mathbf{a} \\ \mathbf{a} \cdot \mathbf{r}_{\alpha \beta} & \mathbf{a} & -\mathbf{a} \\ r_{\alpha \beta}^{k} & k r_{\alpha \beta}^{k-2} \mathbf{r}_{\alpha \beta} & -k r_{\alpha \beta}^{k-2} \mathbf{r}_{\alpha \beta} \\ r_{\alpha \beta}^{k} \mathbf{r}_{\alpha \beta} & (k+3) r_{\alpha \beta}^{k} & -(k+3) r_{\alpha \beta}^{k} \\ \mathbf{r}_{\alpha \beta} \wedge A_{\bar{p}} & (3-p) A_{\bar{p}} & -(3-p) A_{\bar{p}} \\ \mathbf{r}_{\alpha \beta} \cdot \mathbf{r}_{\alpha \gamma} & \mathbf{r}_{\alpha \gamma} & -\left(\mathbf{r}_{\alpha \beta}+\mathbf{r}_{\alpha \gamma}\right),\end{array}$

where $k=0, \pm 1, \pm 2, \pm 3, \ldots$.

Laplace's expansion rule for the inner product of two $p$-blades is given by ${ }^{19}$

$$
\begin{aligned}
\left(\mathbf{a}_{p} \wedge\right. & \left.\cdots \wedge \mathbf{a}_{1}\right) \cdot\left(\mathbf{b}_{1} \wedge \cdots \wedge \mathbf{b}_{p}\right) \\
= & \sum_{k=1}^{p}(-1)^{k+1}\left(\mathbf{a}_{1} \cdot \mathbf{b}_{k}\right)\left(\mathbf{a}_{p} \wedge \cdots \wedge \mathbf{a}_{2}\right) \\
& \cdot\left(\mathbf{b}_{1} \wedge \cdots \wedge \check{\mathbf{b}}_{k} \wedge \cdots \wedge \mathbf{b}_{p}\right),
\end{aligned}
$$

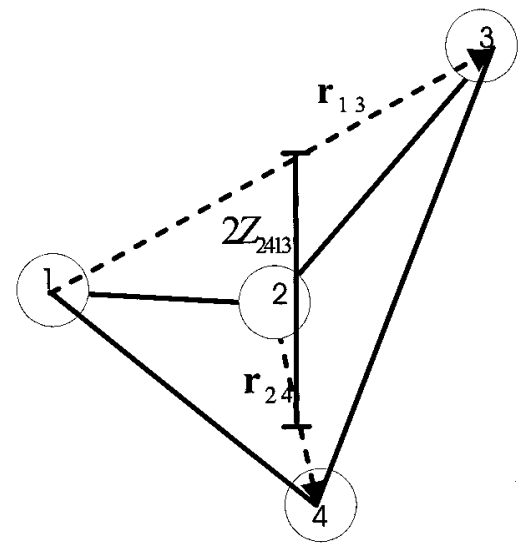

FIG. 7. Ring puckering $Z_{2413}$.

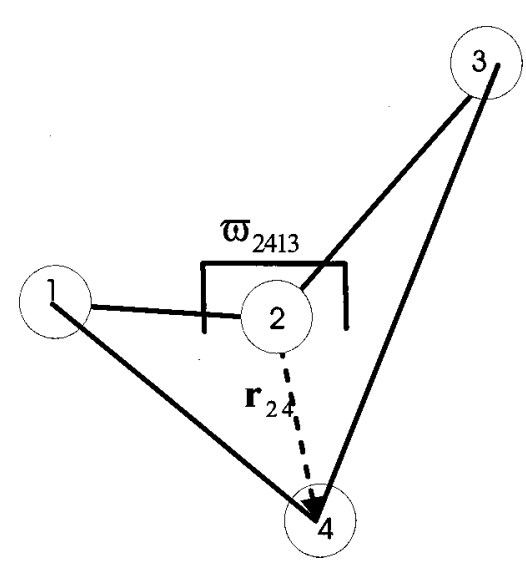

FIG. 8. Diagonal book angle $\varpi_{2413}$. 
where $\check{\mathbf{b}}_{k}$ means that the vector $\mathbf{b}_{k}$ is omitted from the product. Analogously, the expansion rule for the inner product of a vector $\mathbf{a}$ and a $p$-blade $\mathbf{b}_{1} \wedge \cdots \wedge \mathbf{b}_{p}$ is given by

$$
\begin{aligned}
\mathbf{a} \cdot\left(\mathbf{b}_{1} \wedge \cdots \wedge \mathbf{b}_{p}\right)= & \sum_{k=1}^{p}(-1)^{k+1} \mathbf{a} \\
& \cdot\left(\mathbf{b}_{1} \wedge \cdots \wedge \check{\mathbf{b}}_{k} \wedge \cdots \wedge \mathbf{b}_{p}\right) .
\end{aligned}
$$

\section{APPENDIX B: TAYLOR SERIES, WILSON'S METHOD, AND GRADIENTS AT THE REFERENCE CONFIGURATION}

The Taylor expansion of the internal coordinate $q_{i}$ at the point $\mathbf{x}_{\alpha}+\mathbf{a}_{\alpha}$ is ${ }^{19,21}$

$q_{i}\left(\mathbf{x}_{\alpha}+\mathbf{a}_{\alpha}\right)=q_{i}\left(\mathbf{x}_{\alpha}\right)+\mathbf{a}_{\alpha} \cdot \nabla_{\alpha} q_{i}\left(\mathbf{x}_{\alpha}\right)+\frac{\left(\mathbf{a}_{\alpha} \cdot \boldsymbol{\nabla}_{\alpha}\right)^{2}}{2 !} q_{i}\left(\mathbf{x}_{\alpha}\right)+\cdots$

in terms of the position $\mathbf{x}_{\alpha}$ of the nucleus $\alpha$. This is valid for any point $\mathbf{x}_{\alpha}+\mathbf{a}_{\alpha}$. In the infinitesimal limit [i.e., when ( $\mathbf{a}_{\alpha}$ $\cdot \mathbf{u})^{2} \rightarrow 0$ for any unit vector $\mathbf{u}$ ], Eq. (B1) simplifies to

$$
q_{i}\left(\mathbf{x}_{\alpha}+d \mathbf{a}_{\alpha}\right)=q_{i}\left(\mathbf{x}_{\alpha}\right)+d \mathbf{a}_{\alpha} \cdot \nabla_{\alpha} q_{i}\left(\mathbf{x}_{\alpha}\right) .
$$

The infinitesimal displacement is implied by the notation $d \mathbf{a}_{\alpha}$. Furthermore, if $d \mathbf{a}_{\alpha}$ is collinear with $\nabla_{\alpha} q_{i}\left(\mathbf{x}_{\alpha}\right)$, the inner product in Eq. (B2) can be replaced with the geometric product, and

$$
q_{i}\left(\mathbf{x}_{\alpha}+d \mathbf{s}_{\alpha}^{\left(q_{i}\right)}\right)=q_{i}\left(\mathbf{x}_{\alpha}\right)+d \mathbf{s}_{\alpha}^{\left(q_{i}\right)} \nabla_{\alpha} q_{i}\left(\mathbf{x}_{\alpha}\right),
$$

where $d \mathbf{s}_{\alpha}^{\left(q_{i}\right)}$ is an infinitesimal displacement vector to the direction of the gradient $\nabla_{\alpha} q_{i}\left(\mathbf{x}_{\alpha}\right)$. Hence, in the infinitesimal limit, and at the direction of the gradient,

$$
d \mathbf{s}_{\alpha}^{\left(q_{i}\right)} \nabla_{\alpha} q_{i}\left(\mathbf{x}_{\alpha}\right)=q_{i}\left(\mathbf{x}_{\alpha}+d \mathbf{s}_{\alpha}^{\left(q_{i}\right)}\right)-q_{i}\left(\mathbf{x}_{\alpha}\right) .
$$

By multiplying both sides of Eq. (B4) by $\mathbf{u}_{d \mathbf{s}_{\alpha}}\left(q_{i}\right)$ $=d \mathbf{s}_{\alpha}^{\left(q_{i}\right)} /\left|d \mathbf{s}_{\alpha}^{\left(q_{i}\right)}\right|$, the result

$$
\boldsymbol{\nabla}_{\alpha} q_{i}\left(\mathbf{x}_{\alpha}\right)=\frac{d q_{i}}{d s_{\alpha}^{\left(q_{i}\right)}} \mathbf{u}_{d \mathbf{s}_{\alpha}^{\left(q_{i}\right)}}
$$

follows, where $d q_{i}=q_{i}\left(\mathbf{x}_{\alpha}+d \mathbf{s}_{\alpha}^{\left(q_{i}\right)}\right)-q_{i}\left(\mathbf{x}_{\alpha}\right)$ and $d s_{\alpha}^{\left(q_{i}\right)}$ $=\left|d \mathbf{s}_{\alpha}^{\left(q_{i}\right)}\right|$.

Equation (B5) is the mathematical content of Wilson's method. However, it is better to write the result given in Eq. (B5) as

$$
\left.\nabla_{\boldsymbol{\alpha}} q_{i}\right|_{\mathbf{q}^{(e)}}=\frac{d q_{i}}{d s_{\alpha}^{\left(q_{i}\right)}} \mathbf{u}_{d \mathbf{s}_{\alpha}^{\left(q_{i}\right)}}
$$

because in practice the direction of the gradient is deduced geometrically at some configuration $\mathbf{q}^{(e)}=\left\{q_{1}^{(e)}, q_{2}^{(e)}, \ldots\right\}$, and the nucleus $\alpha$ is displaced by an infinitesimal amount $d s_{\alpha}^{\left(q_{i}\right)}$ to this direction $\mathbf{u}_{d \mathbf{s}}{ }_{\alpha}^{\left(q_{i}\right)}=\left.\left(\nabla_{\alpha} q_{i} /\left|\nabla_{\alpha} q_{i}\right|\right)\right|_{\mathbf{q}^{(e)} \text {. The } d q_{i}}$ is the infinitesimal change in the coordinate $q_{i}$ resulting from the infinitesimal displacement of the nucleus $\alpha$. These results are highlighted in the following subsections.

It should also be mentioned that the internal displacement coordinates

$$
\begin{aligned}
\Delta q_{i}\left(\mathbf{a}_{1}, \mathbf{a}_{2}, \ldots\right)= & q_{i}\left(\mathbf{x}_{1}+\mathbf{a}_{1}, \mathbf{x}_{2}+\mathbf{a}_{2}, \ldots\right) \\
& -q_{i}\left(\mathbf{x}_{1}, \mathbf{x}_{2}, \ldots\right)
\end{aligned}
$$

are defined in Ref. 1 by the many variable version of Eq. (B2) as

$$
\Delta q_{i}=\sum_{\alpha}^{N} \mathbf{a}_{\alpha} \cdot \nabla_{\alpha} q_{i}\left(\mathbf{x}_{1}, \mathbf{x}_{2}, \ldots\right) .
$$

Equation (B8) is a linear approximation of Eq. (B7), and they are equal only for the infinitesimal displacements $d \mathbf{a}_{\alpha}$. For example, the coordinate called the "bond length displacement $\Delta r_{\alpha \beta}$ " in Ref. 1, does not equal to the change in the bond length $r_{\alpha \beta}$, except in the case that nuclei $\alpha$ and $\beta$ are displaced by an infinitesimal amount. By expressing the displacement vector $\mathbf{a}_{\alpha}$ of Eq. (B8) in some orthonormal basis $\left\{\mathbf{u}_{1}, \mathbf{u}_{2}, \mathbf{u}_{3}\right\}$ as $\mathbf{a}_{\alpha}=\Sigma_{k}^{3} a_{\alpha_{k}} \mathbf{u}_{k}$, these linearly approximated displacement coordinates can be written as $\Delta q_{i}$ $=\Sigma_{\alpha}^{N} \sum_{k}^{3} B_{i, \alpha_{k}} a_{\alpha_{k}}$, where the $B$-matrix elements are given as $B_{i, \alpha_{k}}=\mathbf{u}_{k} \cdot \nabla_{\alpha} q_{i}\left(\mathbf{x}_{1}, \mathbf{x}_{2}, \ldots\right)$.

\section{Simple example: Valence angle bending}

The most efficient way to increase the valence angle $\theta_{\beta \alpha \gamma}$ by displacing the nucleus $\beta$ is (by intuition) to the direction perpendicular to $\mathbf{r}_{\alpha \beta}$ outwards. This is the direction of the gradient $\mathbf{u}_{d \mathbf{s}_{\beta}^{\left(\theta_{\beta \alpha \gamma}\right)}}=\left(\boldsymbol{\nabla}_{\beta} \theta_{\beta \alpha \gamma} /\left|\nabla_{\beta} \theta_{\beta \alpha \gamma}\right|\right)$, and in the terms of bond vectors it reads as

$$
\mathbf{u}_{d \mathbf{s}_{\beta}^{\left(\theta_{\beta \alpha \gamma}\right)}}=\frac{\mathbf{u}_{\mathbf{r}_{\alpha \beta}} \cos \theta_{\beta \alpha \gamma}-\mathbf{u}_{\mathbf{r}_{\alpha \gamma}}}{\sin \theta_{\beta \alpha \gamma}}
$$

which fortunately is true for any configuration $0<\theta_{\beta \alpha \gamma}<\pi$. The displacement $d s_{\beta}^{\left(\theta_{\beta \alpha \gamma}\right)}$ of the nucleus $\beta$ to the direction $\mathbf{u}_{d \mathbf{s}^{(}{ }_{\beta}}\left(\theta_{\beta \gamma}\right)$ produces a change $d \theta_{\beta \alpha \gamma}$ in $\theta_{\beta \alpha \gamma}$, where

$$
\sin \left(d \theta_{\beta \alpha \gamma}\right)=\frac{d s_{\beta}^{\left(\theta_{\beta \alpha \gamma}\right)}}{r_{\alpha \beta}} \approx d \theta_{\beta \alpha \gamma} .
$$

The latter equality is exact in the case of an infinitesimal displacement, but it would not hold in the case of a unit displacement. Hence, using Eq. (B6), or in this case, Eq. (B5),

$$
\boldsymbol{\nabla}_{\beta} \theta_{\beta \alpha \gamma}=\frac{\mathbf{u}_{\mathbf{r}_{\alpha \beta}} \cos \theta_{\beta \alpha \gamma}-\mathbf{u}_{\mathbf{r}_{\alpha \gamma}}}{r_{\alpha \beta} \sin \theta_{\beta \alpha \gamma}}
$$

which agrees with the result in Eq. (54) derived by using geometric algebra.

\section{Not so simple example: Out-of-plane bending}

In this subsection, I try to derive the gradient $\nabla_{\beta} \varphi_{a \beta \gamma \kappa}$ by using Wilson's method at the planar configuration. At the planar configuration, the most efficient way to increase volume of the parallelepiped spanned by the bond vectors by displacing the nucleus $\beta$ is (by intuition) to the direction perpendicular to the original plane $\mathbf{r}_{\alpha \gamma} \wedge \mathbf{r}_{\alpha \kappa}$. This is the di-

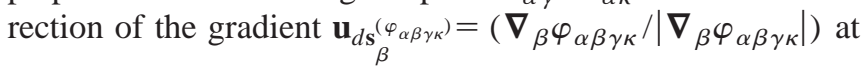
the planar configuration, and in the terms of bond vectors it reads as 


$$
\mathbf{u}_{\left.d \mathbf{s}_{\beta}^{(}{ }_{\alpha \beta \gamma \kappa}\right)}=\frac{\mathbf{r}_{\alpha \gamma} \times \mathbf{r}_{\alpha \kappa}}{\left|\mathbf{r}_{\alpha \gamma} \times \mathbf{r}_{\alpha \kappa}\right|}=\frac{\mathbf{r}_{\alpha \gamma} \times \mathbf{r}_{\alpha \kappa}}{r_{\alpha \gamma} r_{\alpha \kappa} \sin \theta_{\gamma \alpha \kappa}} .
$$

The infinitesimal displacement $d s_{\beta}^{\left(\varphi_{\alpha \beta \gamma \kappa}\right)}$ of the nucleus $\beta$ to the direction $\mathbf{u}_{d \mathbf{s}^{\left(\varphi_{\alpha \beta \gamma \kappa}\right)}}$ produces a change

$$
d \varphi_{\alpha \beta \gamma \kappa}=\frac{\sin \theta_{\gamma \alpha \kappa} d s_{\beta}^{\left(\varphi_{\alpha \beta \gamma \kappa}\right)}}{r_{\alpha \beta}}
$$

in $\varphi_{\alpha \beta \gamma \kappa}$. Hence, using Eq. (B6),

$$
\left.\nabla_{\beta} \varphi_{\alpha \beta \gamma \kappa}\right|_{\varphi_{\alpha \beta \gamma \kappa}=0}=\frac{\mathbf{r}_{\alpha \gamma} \times \mathbf{r}_{\alpha \kappa}}{r_{\alpha \beta} r_{\alpha \gamma} r_{\alpha \kappa}} .
$$

However, this is only a part of the true gradient; the term $-\varphi_{\alpha \beta \gamma \kappa}\left(\mathbf{r}_{\alpha \beta} / r_{\alpha \beta}^{2}\right)$ is missing. This omission is caused by Eq. (B12), which is valid only at the planar configuration. This example clearly shows that the success of Wilson's method may crucially depend on the reference configuration selected. Had the bent instead of planar reference configuration used, the correct gradient could have been obtained (at least in principle, if not in practice-the direction of greatest change is not so obvious in the bent case). Similarly, the "s-vectors" for the bent configuration give the correct gradients for the angle between a bond and a plane defined by two bonds ${ }^{1}$ even at the planar reference configuration.

${ }^{1}$ E. B. Wilson, J. C. Decius, and P. C. Cross, Molecular Vibrations (Dover, New York, 1980).
${ }^{2}$ S. Califano, Vibrational States (Wiley, New York, 1976).

${ }^{3}$ L. Halonen, Adv. Chem. Phys. 104, 41 (1998).

${ }^{4}$ T. Lukka, E. Kauppi, and L. Halonen, J. Chem. Phys. 102, 5200 (1995).

${ }^{5}$ L. Halonen, J. Chem. Phys. 106, 831 (1997).

${ }^{6}$ L. Halonen, J. Chem. Phys. 106, 7931 (1997).

${ }^{7}$ E. Kauppi and L. Halonen, J. Phys. Chem. 94, 5779 (1990).

${ }^{8}$ E. Kauppi and L. Halonen, J. Chem. Phys. 96, 2933 (1992).

${ }^{9}$ E. Venuti, L. Halonen, and R. G. Della Valle, J. Chem. Phys. 110, 7339 (1999).

${ }^{10}$ V. Hänninen, M. Horn, and L. Halonen, J. Chem. Phys. 111, 3018 (1999).

${ }^{11}$ J. Pesonen and L. Halonen (unpublished results).

${ }^{12}$ A. B. MacCoy and E. L. Sibert III, J. Chem. Phys. 95, 3476 (1991).

${ }^{13}$ E. L. Sibert III, J. Hynes, and W. Reinhardt, J. Phys. Chem. 87, 2032 (1983).

${ }^{14}$ D. Swanton and B. Henry, J. Chem. Phys. 86, 4801 (1987).

${ }^{15}$ T. Carrington, J. Chem. Phys. 86, 2207 (1986).

${ }^{16}$ A. R. Hoy, G. Strey, and I. M. Mills, Mol. Phys. 24, 1265 (1972).

${ }^{17}$ B. Podolsky, Phys. Rev. 32, 812 (1928).

${ }^{18}$ G. Gruber, Int. J. Theor. Phys. 6, 31 (1972).

${ }^{19}$ D. Hestenes, New Foundations for Classical Mechanics (Reidel, Dordrecht, 1986).

${ }^{20}$ D. Hestenes and G. Sobczyk, Clifford Algebra to Geometric Calculus (Reidel, Dordrecht, 1984).

${ }^{21}$ D. Hestenes, Synopsis of Geometric Algebra (http:// modelingnts.la.asu.edu/pdf/NFMPchapt1.pdf, 1998).

${ }^{22}$ D. Hestenes, Geometric Calculus (http://modelingnts.la.asu.edu/pdf/ NFMPchapt2.pdf, 1998).

${ }^{23}$ T. G. Vold, Am. J. Phys. 61, 491 (1993).

${ }^{24}$ T. G. Vold, Am. J. Phys. 61, 505 (1993).

${ }^{25}$ J. Laane, M. A. Harthcock, P. M. Killough, L. E. Bauman, and J. M. Cooke, J. Mol. Spectrosc. 91, 286 (1982).

${ }^{26}$ A. Csaszar and N. Handy, Mol. Phys. 86, 959 (1995).

${ }^{27}$ R. Malhiot and S. Ferigle, J. Chem. Phys. 23, 30 (1955). 